\title{
LKB1 Loss Upregulates ALKBH5 and Contributes to Aggressive Phenotypes of KRAS Mutated Lung Cancer
}

\section{Donghong Zhang}

Georgia State University

\section{Jinfeng Ning}

Harbin Medical University Third Hospital: Tumor Hospital of Harbin Medical University

Imoh Okon

Georgia State University

Xiaoxu Zheng

Georgia State University

\section{Ganesh Satyanarayana}

Georgia State University

\section{Ping Song}

Georgia State University

\section{Shidong Xu}

Harbin Medical University Third Clinical College: Tumor Hospital of Harbin Medical University

Ming-Hui Zou ( $D$ mzou@gsu.edu )

Georgia State University

\section{Research}

Keywords: LKB1, KRAS, lung cancer, ALKBH5, RNA methylation, DNA methylation

Posted Date: November 18th, 2020

DOl: https://doi.org/10.21203/rs.3.rs-105982/v1

License: (1) (1) This work is licensed under a Creative Commons Attribution 4.0 International License. Read Full License 


\section{Abstract}

Background: Oncogenic KRAS mutations combined with loss of the LKB1 tumor-suppressor gene (KL) are strongly associated with aggressive forms of lung cancer. N6-methyladenosine (m6A) in mRNA is a crucial epigenetic modification that controls cancer self-renewal and progression. However, the function, regulation and mechanism of $\mathrm{m} 6 \mathrm{~A}$ in this aggressive phenotypes remain largely unclear.

Methods: The clinic-pathological role of m6A was evaluated in a cohort of lung cancer tissues and further validated by public databases and integrating bioinformatics analyses. We examined the upstream and downstream regulation of ALKBH5 (AlkB family member 5 , an m6A demethylase) using quantitative realtime PCR, western blot, bisulfite genome sequencing, luciferase reporter assay, methylated DNA immunoprecipitation, m6A-RNA Immunoprecipitation (m6A-RIP) and m6A-seq data analysis.

Results: We found that LKB1 loss decreased m6A levels and correlated with disease progression and poor survival for KRAS mutant lung cancer patients. The effect on m6A levels and the disease progression and survival was mediated by increasing levels of ALKBH5. LKB1 inactivation increased ALKBH5 transcription in multiple KRAS mutated tumor types, including colon, pancreas, and lung. Conversely, LKB1 overexpression decreased DNA methylation of the CTCF-binding motif on the ALKBH5 promoter, which enhanced CTCF binding and inhibited histone modifications, including H3K4me3, H3K9ac, and H3K27ac. ALKBH5 demethylation of m6A stabilized oncogenic drivers, such as SOX2, SMAD7, and MYC, through a pathway dependent on YTHDF2, an m6A reader protein.

Conclusions: Loss of LKB1 in KRAS mutated cancers promoted ALKBH5 transcription, decreased m6A levels, and increased the stability of $\mathrm{m} 6 \mathrm{~A}$ target oncogenes, thus contributing to aggressive phenotypes of KRAS mutated lung cancer.

\section{Background}

Recent global cancer statistics confirm that lung cancer is a commonly diagnosed malignancy and a leading cause of cancer-related deaths in both men and women [1]. Non-small cell lung cancer (NSCLC) accounts for about $85 \%$ of all lung cancers, and is not sensitive to most available treatment options. Approximately $10 \%$ of NSCLC cases involve concurrent KRAS and LKB1 mutations (KL) [2-4]. These cases are often more aggressive in terms of metastatic spread and drug resistance [5-9] than those with either KRAS or LKB1 mutation. However, the underlying molecular basis of these aggressive clinical behaviors is obscure.

Epigenetic modifications, including DNA/RNA methylation and histone methylation/ acetylation, have important roles in carcinogenesis [10]. KL cancer cells have higher levels of S-adenylmethionine (SAM) synthesis and DNA methylation [11]. SAM increases substrate supply to DNA and histone enzymes, such as DNMT1 and EZH2. Elevated SAM levels may also fuel RNA methylation [12], suggesting that KLmutations mediate a distinct form of epigenetic dysregulation. Nevertheless, reports are lacking on the regulation and role of RNA methylation in $\mathrm{KL}$ cancer cells. 
N6-Methyladenosine (m6A) mainly occurs at the consensus motif of GG ${ }^{\mathrm{m} 6} \mathrm{ACC}$, and is the most prevalent internal chemical modification of mRNAs in eukaryotes [13]. Functionally, the reversible m6A modification of mRNAs is critical to cancer self-renewal and malignancy of several tumors [14-17], including glioblastoma [18], acute myeloid leukemia [19], hepatocellular carcinoma [20], and breast cancer [21]. In this regard, investigation of the landscapes and functions of m6A modifications is an emerging research frontier known as RNA epigenetics or epitranscriptomics. AlkB homolog 5 (ALKBH5), a demethyltransferase of $\mathrm{m} 6 \mathrm{~A}$, is more highly expressed in most tissues than other m6A modulators (8). Alkbh5 deficiency leads to compromised spermatogenesis in mice, and displays widespread mRNA methylation and global RNA instability [22]. Most recently, ALKBH5 was found to have oncogenic roles in glioblastoma and breast cancer cells $(17,20)$, suggesting it contributes to mRNA m6A methylation in cancer.

This study investigated the roles and underlying mechanisms of m6A modulation in aggressive $\mathrm{KL}$ mutant lung cancer cells. We found that loss of LKB1 activity promotes ALKBH5 transcription via DNA methylation and stabilization of m6A target oncogenes in KRAS mutant cancers, which may influence how vulnerable the cancer is to therapy.

\section{Materials And Methods}

Human lung cancer specimens and cell lines

We obtained fresh and paraffin-embedded lung cancer specimens from 72 patients who underwent lung cancer surgery between January 2016 and September 2019 at the Cancer Hospital of Harbin University Medical College, China. Clinical characteristics of patients were retrospectively analyzed. Two pathologists performed blinded histological confirmation by hematoxylin and eosin (H\&E) staining. The clinical pathology variables of lung cancer patients are summarized in Additional table S1. We collected all clinical samples with informed consent according to Health Insurance Portability and Accountability Act (HIPAA)-approved protocols. The use of human patient samples was approved by the Harbin University Medical College Ethics Committee.

MRC-9, H1299, H1650, H1703, H1795, H1792, A549, DLD-1, SW480, Panc-1, and MIA PaCa-2 cell lines were purchased from ATCC, USA. Cells were cultured in medium according to the manufacturer's instructions and grown in a humidified incubator at $5 \% \mathrm{CO}_{2}$. All cell lines were authenticated and confirmed negative for mycoplasma contamination by providers.

RNAi and protein overexpression transfection

To knockdown endogenous gene expression, we purchased si-RNAs targeting human LKB1 (sc-35816), ALKBH5 (sc-93856), and CTCF (sc-35124) from Santa Cruz and transiently transfected these siRNAs into lung cancer cells for $48 \mathrm{~h}$ using Lipofectamine RNAiMAX (Invitrogen). We ordered LKB1 (\#8590) and ALKBH5 (\#38073) overexpressing vectors from Addgene and transfected them into human cancer cells using Lipofectamine 2000 (Invitrogen) for 48 h. Si-RNA-A (sc-37007, Santa Cruz) and c-Flag pcDNA3 
(Plasmid \#20011, Addgene) were served as transfect controls for genes knockdown and overexpression, respectively.

Quantitative reverse transcription polymerase chain reaction (qRT-PCR)

We extracted total cellular and tissue RNA using TRIzol Reagent (Thermo Fisher Scientific, USA) and used $1 \mu \mathrm{g}$ total RNA for reverse transcription using the iScript ${ }^{\mathrm{TM}} \mathrm{cDNA}$ Synthesis Kit (Bio-Rad) according to the manufacturer's instructions. We performed qRT-PCR was performed with $2 \times$ SYBR Green qPCR Master Mix (Bimake, USA) with a CFX96 Touch $^{\text {TM }}$ Real-Time PCR detection system (Bio-Rad Inc. USA). We calculated the relative gene expression using the comparative CT method and $\beta$-actin RNA sequences as a control. Primer sequences are listed in Additional table S2.

Western blot analysis

We lysed total protein from treated cells using RIPA buffer (Cell Signaling Technology) supplemented with Protease Inhibitor Cocktail (ThermoFisher; 78430). We loaded $20 \mu \mathrm{g}$ of protein and separated the sample using $12 \%$ SDS-PAGE. Western blotting was performed as previously described [23], and the primary antibodies included GAPDH (Santa Cruz, sc-137179), LKB1 (Santa Cruz, sc-32245), ALKBH5 (Proteintech, 16837-1-AP), CTCF (Santa Cruz, sc-271474), METTL3 (Abcam, ab195352), METTL14 (Abcam, ab220030), FTO (Abcam, ab126605), WTAP (Proteintech, 60188-1-lg), SOX2 (Cell signaling, \#3579), SMAD7 (Santa Cruz, sc-11392), and MYC (Cell signaling, \#13987). We performed densitometric analyses of band intensity using ImageQuant TL 8.2 image analysis software (GE Healthcare Life Sciences, USA) and GAPDH was as an internal control.

Immunohistochemistry (IHC) staining

We performed IHC analysis of paraffin-embedded lung cancer tissues containing primary tumors and matched normal lung tissues as previously described [24, 25]. In brief, we de-paraffinized and rehydrated human tissue sections to retrieve antigens by microwaving the sections in $10 \mathrm{mM}$ Sodium Citrate $\mathrm{pH}$ 6.0), and then incubating in $1 \%$ hydrogen peroxide to suppress endogenous peroxidase activity. After blocking in 2.5\% horse serum for $1 \mathrm{~h}$, we applied primary antibodies to the slides at 1:500 (anti-LKB1, METTL3, METTL14, WTAP, and FTO antibodies), 1:200 (anti-ALKBH5 antibody), and 1:1000 (anti-m6A and 5-mC antibody) dilutions and incubated at $4^{\circ} \mathrm{C}$ overnight. We stained slides with EnVision + Dual Link System-HRP (Dako) for $1 \mathrm{~h}$ at room temperature, then washed, counter-stained with hematoxylin, dehydrated, treated with xylene, and mounted the samples. Images of stained cells in four random fields were captured by using an optical microscope (Olympus, Japan). Relative protein expression were evaluated by a Histoscore (H-score) system. This semiquantitative approach was calculated as the product of the percentage of positive cells by the staining intensity (graded as: 0 , non-staining; 1 , weak; 2 , median; or 3 , strong using adjacent normal mucosa as the median). Possible scores ranged from 0 to 300. The results were evaluated by two independent pathologists.

Immunofluorescence staining 
We performed immunofluorescence staining on cultured A549 cells transfected with siRNA or pcDNA. After treatment, we fixed cells with $4 \%$ paraformaldehyde (PFA), permeabilized with $0.3 \%$

Triton/phosphate-buffered saline (PBS), blocked in 1\% bovine serum albumin (BSA), and then incubated with the indicated primary antibody for LKB1 (Santa Cruz, sc-32245), ALKBH5 (Proteintech, 16837-1-AP), m6A (Synaptic Systems, 202111), or YAP (Cell signaling, 4912) overnight at $4^{\circ} \mathrm{C}$. The next day, cells were washed and incubated with secondary antibody conjugated to Alexa Fluor 555 (donkey anti-rabbit, Invitrogen) for $1 \mathrm{~h}$ at room temperature. We used DAPI (Sigma) staining to label nuclei. Fluorescence was observed under a Leica SP8 confocal laser scanning microscope.

\section{Luciferase reporter assays}

For the ALKBH5 promoter reporter assay, we generated serial ALKBH5 promoter reporters by PCR amplification and inserted into pGI3-basic plasmid, as we previously reported [23]. We deleted the CTCF region using the Q5 Site-Directed Mutagenesis Kit (NEB) per the manufacturer's protocol. Firefly luciferase activity was used to evaluate the effect of m6A modification on SOX2, SMAD7, and MYC activation. We used the pmirGLO Dual-Luciferase miRNA target expression vector from Promega to construct the reporter plasmid, which contained both a firefly luciferase and a Renilla luciferase. The wild-type SOX2, SMAD7, and MYC reporter plasmid was individually cloned by inserting the fragment containing the m6A peak region after the firefly luciferase coding sequence. We constructed mutant three reporter plasmids by replacing the adenosine bases within the m6A consensus sequences with cytosine. All constructs were confirmed by Sanger sequencing. Nucleotide sequences of primers are in Additional table S2. A549 cells grown in 96-well plates were transfected with reporter vectors and SV-40-Renilla-Luc in the presence of Lipofectamine 2000 Reagent (Invitrogen). After a 24-h transfection, we prepared cell extracts with passive lysis buffer. Luminescence was measured with the Dual-Luciferase Reporter Assay System (Promega), according to manufacturer's instructions. The relative luciferase reporter activities were normalized to that of Renilla. We performed experiments for each vector as biological triplicates with six technical repeats.

Global 5-mC and m6A measurement

We measured total cellular and tissue 5-mC in DNA and m6A in mRNA levels by the EpiQuik m6A RNA Methylation Quantification ELISA kit (Colorimetric) and MethylFlash Methylated DNA 5-mC Quantification Kit (Colorimetric) (Epigentek Group Inc.), respectively. Total genomic DNA and RNA were isolated using the PureLink Genomic DNA Mini Kit and TRIzol Reagent (Thermo Fisher Scientific). We used $200 \mathrm{ng}$ of DNA or RNA for additional global 5-mC and m6A measurement according to the manufacturer's instructions. Measurements were performed in triplicate.

Bisulfite genome sequencing (BGS)

For DNA methylation analysis, we used BGS method, as previously described [26, 27]. Briefly, $500 \mathrm{ng}$ of genomic DNA was bisulfite converted using the BisulFlash DNA Modification Kit (Epigentek) following the manufacturer's instructions. We amplified the fragment containing CTCF peak region of ALKBH5 
promoter using primers listed in Additional table S2. PCR products were purified and cloned into a pCR4 TOPO vector using the TOPO TA Cloning Kit (Thermo Fisher Scientific, Rockford, IL, USA). We isolated and sequenced plasmid DNA from 10 randomly selected clones (Genewiz, Piscataway, NJ, USA).

Methylated DNA immunoprecipitation (MeDIP) analysis

To confirm BGS results, we performed MeDIP using the Methylamp Methylated DNA Capture Kit (EpiGentek) according to manufacturer's instructions. Briefly, we extracted cellular and tissue chromatin DNA and digested to approximately $150-700$ bp using a micrococcal nuclease (CST). The fragmented DNA was immunoprecipitated with anti-5-mC (Abcam, ab10805) at room temperature for $2 \mathrm{~h}$. After washing and purifying the DNA, we quantified the methylation status using qPCR. Primers are list in Additional table $\mathbf{S}$.

m6A-RNA Immunoprecipitation (m6A-RIP)

We extracted total RNA from treated A549 cells or tumor tissues, and incubated with DNase according to the TURBO DNA-free TM Kit (ThermoFisher) protocol to avoid DNA contamination. Then, we chemically fragmented $1 \mu \mathrm{g} / \mu \mathrm{l}$ RNA into $\sim 100 \mathrm{nt}$ size and incubated with m6A antibody to immunoprecipitate according to the standard protocol for the EpiMark N6-Methyladenosine Enrichment Kit (NEB).

Enrichment of m6A containing mRNA was then analyzed using qRT-PCR. Primers targeting m6A-enriched regions of SOX2, SMAD7, and MYC are listed in Additional table S2.

Cell proliferation and migration assay

For cell colony formation assay, A549 or H1792 cells were transfected with siRNA or pcDNA for 24-h and seeded into 6 -well plates (500/well). After 1 week, we fixed formed colonies and stained with $0.1 \%$ crystal violet in $20 \%$ methanol, and counted colonies consisting of at least 50 cells. We calculated relative cloning numbers and created plots using GraphPad Prism 7.0 (GraphPad Software, Inc.). For the migration assay, we re-suspended $5 \times 10^{5}$ cells in Opti-MEM Reduced Serum Media (Invitrogen) and seeded into the upper chamber of a transwell apparatus $(0.8 \mu \mathrm{m}, \mathrm{BD}$ Biosciences), and added complete medium to the bottom chamber to provide chemoattractants for migration. After $24 \mathrm{~h}$, we gently wiped away cells remaining on the upper side of the membrane, and fixed cells that had migrated to the lower side of the membranes with methanol and stained with $1 \%$ crystal violet. To count migrated cells, we captured images of stained cells in five random fields by using an optical microscope (Olympus, Japan) and counted samples in triplicate.

Bioinformatics assay from database

The differentially expressed genes (DEGs) of m6A modulators (METTL3, METTL14, WTAP, FTO, and ALKBH5) and readers (YTHDF1, YTHDF2, YTHDF3, YTHDC1, and YTHDC2) between KL and K-lung cancer cell lines or tissues from the databases of the Cancer Genome Atlas (TCGA, https://portal.gdc.cancer.gov/), Mouse Tumor Biology (MTB, 
http://tumor.informatics.jax.org/mtbwi/index.do), and Cancer Cell Line Encyclopedia (CCLE, https://portals.broadinstitute.org/ccle/home). The RPKM values for each tissue and cell line were obtained from the CCLE repository. Then we compared the differentially expressed genes between $\mathrm{K}$ and $\mathrm{KL}$-lung cancers in above three databases.

m6A-seq data analysis

Based on the NCBI-GEO database (GEO: GSE76367), we determined the number of m6A-seq fragments mapped to each gene using HT-SEq. We overlapped the core m6A peaks based on functional m6A signal enriched around the stop codon (-5kbp + $5 \mathrm{kbp})$ of mRNAs and canonical m6A peaks $[28,29]$. Then, core m6A peaks were subjected to functional enrichment analysis by Ingenuity Pathway Analysis (IPA) (http://www.qiagen. com/ingenuity) and KEGG pathway (https://www.genome.jp/kegg/).

\section{Statistics}

All experiments were repeated at least three times, unless otherwise stated in the figure legend. We performed statistical analyses using the SPSS v20.0 software (SPSS Inc., Chicago, IL), and used Student's t-test (two-tailed) or one-way ANOVA analysis followed by Tukey's, Sidak's, or Bonferroni test to assess statistical significance between or among groups. The relationship between ALKBH5 mRNA expression and DNA methylation, as well as between ALKBH5 and LKB1 mRNA expression, were analyzed by Pearson correlation coefficient. We calculated the survival rate using the log-rank (MantelCox) test. Normality was assumed and variance was compared between or among groups. All numerical data were presented as mean \pm standard deviation (SD) and a p-value of $<0.05$ was considered significant.

\section{Results}

\section{Reduced m6A level was associated with aggressive KL lung cancer}

We first investigated the association between m6A RNA modification and aggressive KL lung cancer. IHC staining showed the reduced m6A level by loss of LKB1 in patients with Kras mutation. And, the lowest of m6A level was found in KL tumor tissues when compared to wild type (WT), L, or $K$ tumor tissues (Fig. 1A, B). The m6A level was inversely correlated with TNM (Tumor, Node and Metastasis) and clinical stage, whereas positively with tumor differentiation in Kras mutant patients, but not in those with Kras wildtype (Fig. 1C). In addition, we found lower m6A level in $\mathrm{K}$ specimens that were null for Thyroid transcription factor 1 (TTF1), a positive prognostic feature (Fig. 1D) [30]. Thus, reduced m6A modification is associated with aggressive $\mathrm{KL}$ lung cancer.

\section{Loss of LKB1 enhanced ALKBH5 responses for m6A reduction in $\mathrm{K}$ lung cancer}


To investigate how $\mathrm{m} 6 \mathrm{~A}$ is regulated in $\mathrm{KL}$ lung cancer, we compared the expression of proteins that act as the m6A writer complex (METTL3, METTL14, and WTAP) and erasers (ALKBH5 and FTO). IHC staining showed that only ALKBH5 expression was higher in KL than that of in $K$ tumor tissues (Fig. 2A, B). ALKBH5 protein expression was negatively correlated with m6A level, in contrast to positive correlation with the TNM and clinical stage in K lung cancer patients (Fig. 2C). However, LKB1 loss alone was not sufficient to change m6A level or ALKBH5 expression or their relationship with aggressive tumor phenotypes (Additional Fig S1 A-D).

To confirm observations of clinical specimens, we screened for the differential expressions of m6A mediators and readers based on database queries. Analysis of TCGA, MTB [8, 31], and CCLE databases revealed that ALKBH5 mRNA expression was consistently higher in $\mathrm{KL}$ cancer tissues or cells, and negatively correlated with LKB1 expression (Fig. 2D, Additional Fig. S2A-D). Furthermore, elevated ALKBH5 expression correlated with poor prognosis for patients with KRAS mutation (Fig. 2E). Additionally, ALKBH5 had the highest basal mRNA expression in human and mouse lung cancer tissues and cell lines (Additional Fig.S2A-C). Taken together, these findings indicate that ALKBH5, a major regulator of m6A modification, contributes to aggressive phenotypes of $\mathrm{KL}$ lung tumors.

To investigate whether LKB1 loss affected ALKBH5 and m6A modification, we screened several lung cancer cell lines and categorized them based on KRAS mutation and LKB1 expression status. A549KL

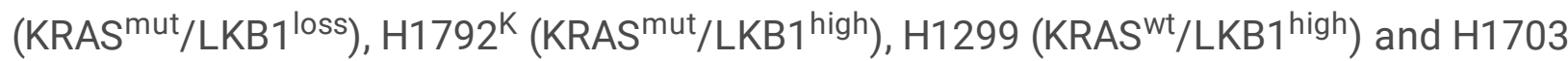
$\left(K_{R A S}{ }^{w t} / L K B 1^{\text {low }}\right.$ ) were selected from each category for further analyses (Additional Fig. S3A). We then generated the loss of LKB1 function by siRNA-LKB1 transfection in $\mathrm{H} 1299$ and $\mathrm{H} 1792$ cells, while gain-offunction by LKB1 overexpression in H1703 and A549 cells. As expected, LKB1 expression negatively regulated m6A levels in cells with KRAS mutation, but not with KRAS wildtype (Fig. 2F). Consistent with clinical data, western blot and qRT-PCR assays showed that ALKBH5 was also the only modulator negatively regulated by LKB1 in KRAS ${ }^{\text {mut }}$ cells, but was unaffected in KRAS ${ }^{\text {wt }}$ cells (Fig. 2G, Additional Fig S3B-E). These observations were supported by immunofluorescence staining that demonstrated negligible ALKBH5 signal in LKB1-overexpression A549 cells (Fig. 2H). Exogenous ALKBH5 expression blocked m6A staining in the presence of LKB1 (Fig. 2I). Furthermore, LKB1 expression negatively correlated with ALKBH5 expression was also found in KRAS-mutated pancreatic and colorectal cancer cell lines (Additional Fig. S4A-D). Therefore, KRAS mutation and LKB1 expression directly affected global m6A levels via ALKBH5.

\section{ALKBH5 upregulation increased cell proliferation and migration in KL lung cancer cells}

Next, we explored functional relationships between LKB1 and ALKBH5 in KRAS mutant lung cancer cells. First, we established the transient ALKBH5 and/or LKB1 knockdown models in $\mathrm{H} 1792$ cells, as well as overexpression in A549 cells (Fig. 3A). As expected, ALKBH5 could fully release LKB1 repressed m6A levels in both cell lines (Fig. 3B, C). Functionally, ALKBH5 knockdown significantly inhibited cell proliferation, as shown by decreased colony formation in LKB1-silenced H1792 cells (Fig. 3D). We 
observed similar for H1792 cell migration in the transwell assay (Fig. 3E). Conversely, ALKBH5 overexpression promoted cell proliferation and migration in LKB1-transfected A549 cells (Fig. 3F, G). Thus, LKB1 loss correlated with upregulation of ALKBH5, and increased cell proliferation and migration in KRAS mutant cells.

Loss of LKB1 upregulated ALKBH5 via DNA hypermethylation in KRAS mutant cancer cells.

We then explored how LKB1 regulated ALKBH5 through DNA methylation. Consistent with previous studies in pancreatic ductal epithelial cells [11], LKB1 alterations also negatively regulated the global $5 \mathrm{mC}$ DNA methylation in KRAS mutant lung cancer cells (Fig. 4A) but had no effect in KRAS wildtype cells. Specifically, ALKBH5 gene DNA methylation positively correlated with ALKBH5 mRNA expression in KRAS mutant lung, and colorectal cancer cell lines, based on the CLLE database (Additional Fig. S5A-D). Subsequent treatment with 5-azacytidine (5-aza), an inhibitor of DNA methylation, reduced ALKBH5 mRNA and protein expression in a dose-dependent manner in $\mathrm{H} 1792$ and $\mathrm{A} 549$ cells, independent of LKB1 status (Fig. 4B-D). Based on ChIP-sequence analysis from the ENCODE database, we identified a single putative transcriptional repressor, CTCF (CCCTC-binding factor) and several activators, including H3K4me1, H3K4me2, H3K4me3, H3K9ac, and H3K27ac, on the ALKBH5 gene core promoter in A549 cells (Additional Fig. S6). Interestingly, the CTCF peak region was co-located in the CpG island of ALKBH5 promoter (Fig. 4E). Further analysis by MeDIP assay indicated $5 \mathrm{mC}$-methylation on the CTCF peak region was decreased with 5-aza treatment or exogenous LKB1-transfection in A549 cells (Fig. 4F). Lastly, bisulfite sequencing confirmed decreased DNA methylation on the CTCF peak region in 5-aza-treated or LKB1 over-expressed cells (Fig. 4G, H). Thus, the data indicates that LKB1 loss induced DNA hypermethylation, thereby controlling ALKBH5 expression in KRAS mutant cancer cells.

\section{DNA hypermethylation of the CTCF motif is critical for ALKBH5 upregulation.}

To determine whether CTCF directly represses ALKBH5, we used western blot and RT-qPCR. We found that silencing CTCF increased ALKBH5 protein and mRNA expression, and also rescued ALKBH5 repression by LKB1 overexpression (Fig. 5A-C). We next generated serial deletions, including CTCF motif deletion constructs based on the human ALKBH5 promoter, for the luciferase reporter assay. The CTCF-containing construct had lower ALKBH5 promoter activity than constructs that lacked the motif (Fig. 5D). Notably, CTCF motif deletion increased ALKBH5 promoter activity to levels similar to those in constructs without the CTCF motif. This evidence suggests that CTCF directly represses ALKBH5 transcriptional activity.

Next, we found that DNA demethylation by 5-aza treatment or by LKB1 overexpression reduced ALKBH5 promoter activity on the CTCF-containing construct, but had no effect on CTCF-deficient construct (Fig. 5E). Further ChIP-qPCR analysis indicated that histone activators, such as H3K4me1, H3K4me2, H3K4me3, H3K9ac, and H3K27ac, occupy the ALKBH5 gene promoter (Fig. 5F), consistent with ChIP-seq results (Additional Fig. S6). Interestingly, 5-aza treatment or LKB1 overexpression promoted CTCF enrichment on ALKBH5, and also partially prevented enrichment of H3K4me3, H3K9ac, and H3K27ac (Fig. 5H-J). Therefore, loss of LKB1 promoted DNA hyper-methylation in the CTCF peak region, thus preventing CTCF binding and releasing repression of ALKBH5 in KRAS mutant cells. 
ALKBH5 demethylation of m6A increased expression and stability of SOX2, SMAD7, and MYC in a YTHDF2-dependent pathway

To identify downstream targets of ALKBH5 mediated m6A modification in lung cancer, we overlapped 2,605 genes using the canonical m6A motif-enriched gene stop codon region based on m6A-sequence in A549 cells (20) (Additional Fig. S7A). Gene ontology analysis revealed that the 2,605 genes were significantly enriched in gene transcription regulation, mRNA splicing, cell cycle, and mRNA stability (Additional Fig. S7B). KEGG pathway analysis revealed that the overlapping genes were closely associated with Hippo and TGF- $\beta$ pathways (Additional Fig. S7C). Using qRT-PCR, we confirmed that 45.2\% (14/31) of Hippo-Yap pathway genes were directly regulated by ALKBH5 (Additional Fig. S7D, E). Western blot assay further confirmed that ALKBH5 regulated SOX2, SMAD7, and MYC proteins in A549 and $\mathrm{H} 1792$ cells (Fig. 6A, B).

To investigate m6A modifications on SOX2, SMAD7, and c-MYC mRNA, we used firefly luciferase assay (Fig. 6C). We found their activities were significantly less than those of their mutant reporters, indicating that m6A repressed gene activity. Moreover, overexpression of ALKBH5 increased luciferase activities of three m6A WT gene reporters, and also rescued the repressive activity mediated by LKB1-transfection. However, we saw no such effects for m6A mutant gene-fused reporters. Our m6A-RIP-qPCR assay showed significantly higher accumulation of m6A at mRNA fragments of all three genes than for the IgG control. However, this m6A enrichment was reduced by ALKBH5 overexpression. Also, ALKBH5 upregulation inhibited the increased m6A occupancy caused by LKB1 overexpression (Fig. 6D). Given that ALKBH5 overexpression or knockdown did not change the pre-mRNAs of SMAD7 and MYC (Additional Fig S8A-D), we predicted m6A reader protein YTHDF2 regulated their mRNA stability. Interestingly, actinomycin-D induced degradation of SMAD7, SOX2, and MYC mRNAs was partially prevented by silencing YTHDF2 in both A549 and H1792 cells. (Additional Fig. S8E-J). RIP-qPCR assay showed that YTHDF2 also occupied SMAD7, SOX2, and MYC m6A regions, and this enrichment increased in the absence of ALKBH5 (Fig. 6E). Furthermore, YTHDF2 silencing rescued their gene expression upon LKB1 overexpression or ALKBH5 knockdown (Fig. 6F). Thus, ALKBH5-m6A-YTHDF2 signaling prevented SOX2, SMAD7, and MYC mRNA decay.

Confirmation of an axis of "LKB1-DNA methylation-ALKBH5-m6A" in clinical KRAS mutated lung cancer patients.

Next, we validated the underlying regulation of m6A by LKB1 using a panel of clinically relevant lung adenocarcinoma specimens. LKB1 protein expression negatively correlated with global 5-mC DNA methylation in lung cancer tissues with KRAS mutations, but not in tissues with WT KRAS (Additional Fig. S9A, B). Notably, ALKBH5 DNA methylation positively correlated with ALKBH5 mRNA expression and both expression significantly increased with LKB1 deficiency in patients with KRAS mutation (Additional Fig. S9C-E). ALKBH5 protein expression negatively correlated with global m6A level, as well as the m6A levels for SOX2, SMAD7, and MYC genes in KRAS mutant tissues (Additional Fig. S9F-H). Furthermore, m6A levels of these three genes negatively correlated with their mRNA expression (Additional Fig. S9I-K). 


\section{Discussion}

Here, we report that reduced m6A RNA modification increased stability and expression of critical oncogenes and contributed to aggressive cancer phenotypes. Loss of LKB1 specifically enhanced ALKBH5 expression and reduced m6A levels in KRAS mutated cells. LKB1 inactivation could increase the 5-mC DNA methylation of the ALKBH5 promoter, which prevents CTCF binding and releases ALKBH5 suppression. Subsequently, reduced "m6A-YTHDF2" signaling promoted the expression and stability of critical tumor oncogenes, such as SOX2, SMAD7, and MYC. Our findings describe a mechanism of crosstalk between $5 \mathrm{mC}$-DNA and m6A RNA modification in $\mathrm{KL}$ tumors that supports tumorigenic growth and progression (Fig. 7). Our study indicates that LKB1 loss reduced m6A modification by upregulating ALKBH5, which contributes to aggressive tumor progression and poorer outcomes for KL lung cancer.

Aberrant global m6A abundance is increasingly reported in human cancers, and may be associated with cancer progression and clinical outcome [32]. Interestingly, m6A hypomethylation was reported in glioblastoma, bladder cancer, endometrial cancer [33], melanoma, or breast carcinoma, whereas, m6A hypermethylation was found in gastric cancer and hepatocellular carcinoma [34]. Writing and erasing proteins regulate m6A levels, which enable the binding of $\mathrm{m} 6 \mathrm{~A}$ reader proteins and initiate a series of biological functions. For example, ALKBH5 demethylates m6A mRNAs, and modulates mRNA splicing, export and stability. Alkbh5 deficiency leads to aberrant spermatogenesis and apoptosis in mouse testes, likely through regulating genes associated with the p53 network [22]. ALKBH5 often exerts an oncogenic role in GBM, pancreas, cervical, and breast cancer, but acts a tumor-suppressor in leukemia [32]. This twosided role of ALKBH5, might relate with the complex and diverse function of m6A modification, which not only promotes the translation of related mRNAs but also reduces the mRNAs stability by binding with different reader proteins [13-16]. Thus, It would be important to determine how critical role of $\mathrm{m} 6 \mathrm{~A}$ and its regulator in each type of cancer.

Limited literature is available to explain how m6A regulators modulate cancers, although many studies have describes roles for m6A regulators in cell fate and carcinogenesis $[20,35,36]$. This study identifies ALKBH5 as a functional target gene of 5-mC DNA, which controls m6A RNA. Notably, ALKBH5 is one of the top five 5-mC DNA biomarkers that helped distinguish patients with metastatic-lethal prostate cancer [37], suggesting that DNA methylation of ALKBH5 may be a prognostic indicator.

We also identified CTCF as an effective transcriptional suppressor that was highly sensitive to global 5mC DNA methylation and required for ALKBH5 repression. Similarly, we previously showed that DNA hypomethylation facilitated CTCF binding to and suppression of hTERT expression in human endothelium [26]. Recent studies show 5-mC DNA hypermethylation facilitated tumorigenesis in KRAS/LKB1 co-mutated cancer [11]. LKB1 inactivation causes DNA hypermethylation and histone methylation, which facilitates immune escape in KL-mutated lung cancer and represses anti-oncogenic STING [38]. Consistent with those previous findings, our work indicates that oncogenic ALKBH5 is another target of LKB1 by a similar mechanism. Thus, loss of LKB1 reduces m6A modification also might via the linking of $5 \mathrm{mC}$-DNA and histone modification in $\mathrm{KL}$ cancer. 
Consistent with previous studies, our observations indicate that ALKBH5 is a lung cancer oncogene because it promoted cell proliferation and migration $[18,21]$. Increased ALKBH5 expression predicted poor survival in our analysis. Moreover, we identified three critical oncogenes, SOX2, SMAD7, and MYC, as targets of ALKBH5-mediated m6A modification. Mechanistically, LKB1 loss or ALKBH5 overexpression increased SAMD7, SOX2, and MYC stability via a YTHDF2-dependent mechanism. This result is supported by previously reported PAR-CLIP-Seq data in a Hela cell line [13]. YTHDF2-induced decay of m6A modified genes may be a common pathway across cell types. We also found that YTHDF1 occupied and promoted SAMD7 or MYC mRNA translation in an m6A-dependent manner [39]. Thus, it would be interesting to compare proteomic changes between tumor and non-tumor samples upon METTL3 manipulation.

Notably, LKB1 regulation of ALKBH5 via 5mC-DNA was not limited to KRAS mutant lung cancer, but extended to pancreatic and colorectal cancer, which are the top three causes of cancer death in the United States [40]. Aggressive lung tumorigenesis, tumor progression, and poor prognosis were observed in mice with Kras mutation combined with $L k b 1$ inactivation [8]. This tumor type is largely resistant to both standard-of-care treatments like docetaxel and combination treatment with a MEK inhibitor [5, 41]. Comutations were also associated with an inert tumor immune microenvironment and poor clinical response to immune checkpoint blockade [42-44]. Thus, understanding molecular mechanisms may improve therapeutic strategies for cancer with KRAS and LKB1 co-mutations. Screening chemicals that may regulate $\mathrm{m} 6 \mathrm{~A}$ formation or removal is an effective approach for developing tumor therapeutics. For example, R-2HG, an ALKBH5 and FOT inhibitor, inhibits leukemia cell growth and induces apoptosis in mice [45]. m6A-YTHDF2 inactivity contributes to melanoma progression by enhancing the expression and stability of key immune checkpoint factors, including PD-1, CXCR4, and SOX10[46]. This implies that YTHDF2 modulation could be combined with an anti-PD-1/PD-L1 blockade to improve anticancer immunotherapy. In support of this idea, we identified the ALKBH5-m6A-YHTDF2 axis as a targetable molecular pathway to treat this aggressive cancer.

\section{Conclusions}

In conclusion, our results suggest that LKB1 loss caused epigenetic reprogramming via 5mC-DNA methylation. We also found that histone modification was necessary for ALKBH5 upregulation, which in turn controlled m6A RNA modification for KRAS co-mutated cancers. Our findings and other recent reports $[10,14,15,17]$ highlight the importance of the global m6A modification in cancer. Global m6A modification may selectively activate oncogenic drivers, such as SOX2, SMAD7, and MYC, to promote aggressive tumor phenotypes. Our findings on epigenetic reprogramming indicate new therapeutic approaches to tumors with dual LKB1 and KRAS mutation.

\section{Abbreviations}

m6A, N6-methyladenosine; ALKBH5, AlkB family member 5; NSCLC, Non-small cell lung cancer; IHC, Immunohistochemistry; BGS, Bisulfite genome sequencing; m6A-RIP, m6A-RNA Immunoprecipitation; 
CTCF, CCCTC-binding factor

\section{Declarations}

Ethics approval and consent to participate: This project was approved by the Ethical Committee on Scientific Research of Harbin Medical University Cancer Hospital.

Consent for publication: All human tissue samples were obtained with written informed consent from all subjects.

Competing interests: The authors declare that they have no competing interests.

Funding: This study was funded by the National Cancer Institute (NCI) (CA213022). Dr. Zou is the Eminent Scholar in Molecular and Translational Medicine of the Georgia Research Alliance.

Authors' Contributions: Conception and design: M. Zou, D. Zhang; Development of methodology: D. Zhang, J. Ning I. Okon; Acquisition of data (provided cell lines, acquired and managed patients, provided facilities, etc.): J. Ning, I. Okon, S, Xu, P. Song, M. Zou; Analysis and interpretation of data (e.g., statistical analysis, biostatistics, computational analysis): D. Zhang, J. Ning, I. Okon, X. Zheng, S. Xu, P. Song; Writing, review, and/or revision of the manuscript: D. Zhang, I. Okon, X. Zheng, G. Satyanarayana, P. Song, M. Zou; Administrative, technical, or material support (i.e., reporting or organizing data, constructing databases): D. Zhang, P. Song, M. Zou; Study supervision: M. Zou.

Acknowledgements: Not applicable.

\section{References}

1. Bray F, Ferlay J, Soerjomataram I, Siegel RL, Torre LA, Jemal A. Global cancer statistics 2018: GLOBOCAN estimates of incidence and mortality worldwide for 36 cancers in 185 countries. CA Cancer J Clin. 2018;68(6):394-424.

2. Sanchez-Cespedes M, Parrella P, Esteller M, Nomoto S, Trink B, Engles JM, Westra WH, Herman JG, Sidransky D. Inactivation of LKB1/STK11 is a common event in adenocarcinomas of the lung. Cancer Res. 2002;62(13):3659-62.

3. Matsumoto S, Iwakawa R, Takahashi K, Kohno T, Nakanishi Y, Matsuno Y, Suzuki K, Nakamoto M, Shimizu E. Minna JD and Yokota J. Prevalence and specificity of LKB1 genetic alterations in lung cancers. Oncogene. 2007;26(40):5911-8.

4. Skoulidis F, Byers LA, Diao L, Papadimitrakopoulou VA, Tong P, Izzo J, Behrens C, Kadara H, Parra ER, Canales JR, Zhang J, Giri U, Gudikote J, et al. Co-occurring genomic alterations define major subsets of KRAS-mutant lung adenocarcinoma with distinct biology, immune profiles, and therapeutic vulnerabilities. Cancer Discov. 2015;5(8):860-77. 
5. Calles A, Sholl LM, Rodig SJ, Pelton AK, Hornick JL, Butaney M, Lydon C, Dahlberg SE, Oxnard GR, Jackman DM, Janne PA. Immunohistochemical Loss of LKB1 Is a Biomarker for More Aggressive Biology in KRAS-Mutant Lung Adenocarcinoma. Clin Cancer Res. 2015;21(12):2851-60.

6. Gilbert-Ross M, Konen J, Koo J, Shupe J, Robinson BS, Wiles WGt, Huang C, Martin WD, Behera M, Smith GH, Hill CE, Rossi MR, Sica GL, et al. Targeting adhesion signaling in KRAS, LKB1 mutant lung adenocarcinoma. JCI Insight. 2017;2(5):e90487.

7. Skoulidis F, Goldberg ME, Greenawalt DM, Hellmann MD, Awad MM, Gainor JF, Schrock AB, Hartmaier RJ, Trabucco SE, Gay L, Ali SM, Elvin JA, Singal G, et al. STK11/LKB1 Mutations and PD-1 Inhibitor Resistance in KRAS-Mutant Lung Adenocarcinoma. Cancer Discov. 2018;8(7):822-35.

8. Ji H, Ramsey MR, Hayes DN, Fan C, McNamara K, Kozlowski P, Torrice C, Wu MC, Shimamura T, Perera SA, Liang MC, Cai D, Naumov GN, et al. LKB1 modulates lung cancer differentiation and metastasis. Nature. 2007;448(7155):807-10.

9. Wang Y, Li N, Jiang W, Deng W, Ye R, Xu C, Qiao Y, Sharma A, Zhang M, Hung MC, Lin SH. Mutant LKB1 Confers Enhanced Radiosensitization in Combination with Trametinib in KRAS-Mutant NonSmall Cell Lung Cancer. Clin Cancer Res. 2018;24(22):5744-56.

10. Michalak EM, Burr ML, Bannister AJ, Dawson MA. The roles of DNA, RNA and histone methylation in ageing and cancer. Nat Rev Mol Cell Biol. 2019.

11. Kottakis F, Nicolay BN, Roumane A, Karnik R, Gu H, Nagle JM, Boukhali M, Hayward MC, Li YY, Chen T, Liesa M, Hammerman PS, Wong KK, et al. LKB1 loss links serine metabolism to DNA methylation and tumorigenesis. Nature. 2016;539(7629):390-5.

12. Stojkovic V, Fujimori DG. Radical SAM-Mediated Methylation of Ribosomal RNA. Methods Enzymol. 2015;560:355-76.

13. Wang X, Lu Z, Gomez A, Hon GC, Yue Y, Han D, Fu Y, Parisien M, Dai Q, Jia G, Ren B. Pan T and He C. N6-methyladenosine-dependent regulation of messenger RNA stability. Nature. 2014;505(7481):11720.

14. Pan Y, Ma P, Liu Y, Li W, Shu Y. Multiple functions of m(6)A RNA methylation in cancer. J Hematol Oncol. 2018;11(1):48.

15. Wang S, Sun C, Li J, Zhang E, Ma Z, Xu W, Li H, Qiu M, Xu Y, Xia W, Xu L, Yin R. Roles of RNA methylation by means of $\mathrm{N}(6)$-methyladenosine $(\mathrm{m}(6) \mathrm{A})$ in human cancers. Cancer Lett. 2017;408:112-20.

16. Lin S, Choe J, Du P, Triboulet R, Gregory RI. The m(6)A Methyltransferase METTL3 Promotes Translation in Human Cancer Cells. Mol Cell. 2016;62(3):335-45.

17. Jaffrey $S R$, Kharas MG. Emerging links between $m(6) A$ and misregulated mRNA methylation in cancer. Genome Med. 2017;9(1):2.

18. Zhang S, Zhao BS, Zhou A, Lin K, Zheng S, Lu Z, Chen Y, Sulman EP, Xie K, Bogler O, Majumder S, He C, Huang S. m(6)A Demethylase ALKBH5 Maintains Tumorigenicity of Glioblastoma Stem-like Cells by Sustaining FOXM1 Expression and Cell Proliferation Program. Cancer Cell. 2017;31(4):591-606 e596. 
19. Li Z, Weng H, Su R, Weng X, Zuo Z, Li C, Huang H, Nachtergaele S, Dong L, Hu C, Qin X, Tang L, Wang $\mathrm{Y}$, et al. FTO Plays an Oncogenic Role in Acute Myeloid Leukemia as a N(6)-Methyladenosine RNA Demethylase. Cancer Cell. 2017;31(1):127-41.

20. Chen M, Wei L, Law CT, Tsang FH, Shen J, Cheng CL, Tsang LH, Ho DW, Chiu DK, Lee JM, Wong CC, $\mathrm{Ng}$ IO, Wong CM. RNA N6-methyladenosine methyltransferase-like 3 promotes liver cancer progression through YTHDF2-dependent posttranscriptional silencing of SOCS2. Hepatology. 2018;67(6):2254-70.

21. Zhang C, Samanta D, Lu H, Bullen JW, Zhang H, Chen I, He X, Semenza GL. Hypoxia induces the breast cancer stem cell phenotype by HIF-dependent and ALKBH5-mediated m(6)A-demethylation of NANOG mRNA. Proc Natl Acad Sci U S A. 2016;113(14):E2047-56.

22. Zheng G, Dahl JA, Niu Y, Fedorcsak P, Huang CM, Li CJ, Vagbo CB, Shi Y, Wang WL, Song SH, Lu Z, Bosmans RP, Dai Q, et al. ALKBH5 is a mammalian RNA demethylase that impacts RNA metabolism and mouse fertility. Mol Cell. 2013;49(1):18-29.

23. Zhang D, Xie X, Chen Y, Hammock BD, Kong W, Zhu Y. Homocysteine upregulates soluble epoxide hydrolase in vascular endothelium in vitro and in vivo. Circ Res. 2012;110(6):808-17.

24. Okon IS, Coughlan KA, Zhang C, Moriasi C, Ding Y, Song P, Zhang W, Li G, Zou MH. Protein kinase LKB1 promotes RAB7-mediated neuropilin-1 degradation to inhibit angiogenesis. J Clin Invest. 2014;124(10):4590-602.

25. Wu L, Zhang D, Zhou L, Pei Y, Zhuang Y, Cui W, Chen J. FUN14 domain-containing 1 promotes breast cancer proliferation and migration by activating calcium-NFATC1-BMI1 axis. EBioMedicine. 2019;41:384-94.

26. Zhang D, Sun X, Liu J, Xie X, Cui W, Zhu Y. Homocysteine accelerates senescence of endothelial cells via DNA hypomethylation of human telomerase reverse transcriptase. Arterioscler Thromb Vasc Biol. 2015;35(1):71-8.

27. Zhang D, Wu B, Wang P, Wang Y, Lu P, Nechiporuk T, Floss T, Greally JM, Zheng D, Zhou B. Non-CpG methylation by DNMT3B facilitates REST binding and gene silencing in developing mouse hearts. Nucleic Acids Res. 2017;45(6):3102-15.

28. Linder B, Grozhik AV, Olarerin-George AO, Meydan C, Mason CE, Jaffrey SR. Single-nucleotideresolution mapping of m6A and m6Am throughout the transcriptome. Nat Methods. 2015;12(8):767-72.

29. Wang Y, Li Y, Toth JI, Petroski MD, Zhang Z, Zhao JC. N6-methyladenosine modification destabilizes developmental regulators in embryonic stem cells. Nat Cell Biol. 2014;16(2):191-8.

30. Kim JH, Kim HS, Kim BJ, Han B, Choi DR, Kwon JH. Prognostic Impact of TTF-1 Expression in NonSquamous Non-Small-Cell Lung Cancer: A Meta-Analysis. J Cancer. 2018;9(22):4279-86.

31. Carretero J, Shimamura T, Rikova K, Jackson AL, Wilkerson MD, Borgman CL, Buttarazzi MS, Sanofsky BA, McNamara KL, Brandstetter KA, Walton ZE, Gu TL, Silva JC, et al. Integrative genomic and proteomic analyses identify targets for Lkb1-deficient metastatic lung tumors. Cancer Cell. 2010;17(6):547-59. 
32. Huang H, Weng H, Chen J. m(6)A Modification in Coding and Non-coding RNAs: Roles and Therapeutic Implications in Cancer. Cancer Cell. 2020;37(3):270-88.

33. Liu J, Eckert MA, Harada BT, Liu SM, Lu Z, Yu K, Tienda SM, Chryplewicz A, Zhu AC, Yang Y, Huang JT, Chen SM, Xu ZG, et al. m(6)A mRNA methylation regulates AKT activity to promote the proliferation and tumorigenicity of endometrial cancer. Nat Cell Biol. 2018;20(9):1074-83.

34. Lan Q, Liu PY, Haase J, Bell JL, Huttelmaier S, Liu T. The Critical Role of RNA m(6)A Methylation in Cancer. Cancer Res. 2019;79(7):1285-92.

35. Fu Y, Dominissini D, Rechavi G, He C. Gene expression regulation mediated through reversible $m(6) A$ RNA methylation. Nat Rev Genet. 2014;15(5):293-306.

36. Geula S, Moshitch-Moshkovitz S, Dominissini D, Mansour AA, Kol N, Salmon-Divon M, Hershkovitz V, Peer E, Mor N, Manor YS, Ben-Haim MS, Eyal E, Yunger S, et al. Stem cells. m6A mRNA methylation facilitates resolution of naive pluripotency toward differentiation. Science. 2015;347(6225):1002-6.

37. Zhao S, Geybels MS, Leonardson A, Rubicz R, Kolb S, Yan Q, Klotzle B, Bibikova M, Hurtado-Coll A, Troyer D, Lance R, Lin DW, Wright JL, et al. Epigenome-Wide Tumor DNA Methylation Profiling Identifies Novel Prognostic Biomarkers of Metastatic-Lethal Progression in Men Diagnosed with Clinically Localized Prostate Cancer. Clin Cancer Res. 2017;23(1):311-9.

38. Kitajima S, Ivanova E, Guo S, Yoshida R, Campisi M, Sundararaman SK, Tange S, Mitsuishi Y, Thai TC, Masuda S, Piel BP, Sholl LM, Kirschmeier PT, et al. Suppression of STING Associated with LKB1 Loss in KRAS-Driven Lung Cancer. Cancer Discov. 2019;9(1):34-45.

39. Wang X, Zhao BS, Roundtree IA, Lu Z, Han D, Ma H, Weng X, Chen K, Shi H, He C. N(6)methyladenosine Modulates Messenger RNA Translation Efficiency. Cell. 2015;161(6):1388-99.

40. Rahib L, Smith BD, Aizenberg R, Rosenzweig AB, Fleshman JM, Matrisian LM. Projecting cancer incidence and deaths to 2030: the unexpected burden of thyroid, liver, and pancreas cancers in the United States. Cancer Res. 2014;74(11):2913-21.

41. Meng D, Yuan M, Li X, Chen L, Yang J, Zhao X, Ma W, Xin J. Prognostic value of K-RAS mutations in patients with non-small cell lung cancer: a systematic review with meta-analysis. Lung Cancer. 2013;81(1):1-10.

42. Moro M, Caiola E, Ganzinelli M, Zulato E, Rulli E, Marabese M, Centonze G, Busico A, Pastorino U, de Braud FG, Vernieri C, Simbolo M, Bria E, et al. Metformin Enhances Cisplatin-Induced Apoptosis and Prevents Resistance to Cisplatin in Co-mutated KRAS/LKB1 NSCLC. J Thorac Oncol. 2018;13(11):1692-704.

43. Koyama S, Akbay EA, Li YY, Aref AR, Skoulidis F, Herter-Sprie GS, Buczkowski KA, Liu Y, Awad MM, Denning WL, Diao L, Wang J, Parra-Cuentas ER, et al. STK11/LKB1 Deficiency Promotes Neutrophil Recruitment and Proinflammatory Cytokine Production to Suppress T-cell Activity in the Lung Tumor Microenvironment. Cancer Res. 2016;76(5):999-1008.

44. Schabath MB, Welsh EA, Fulp WJ, Chen L, Teer JK, Thompson ZJ, Engel BE, Xie M, Berglund AE, Creelan BC, Antonia SJ, Gray JE, Eschrich SA, et al. Differential association of STK11 and TP53 with 
KRAS mutation-associated gene expression, proliferation and immune surveillance in lung adenocarcinoma. Oncogene. 2016;35(24):3209-16.

45. Su R, Dong L, Li C, Nachtergaele S, Wunderlich M, Qing Y, Deng X, Wang Y, Weng X, Hu C, Yu M, Skibbe J, Dai Q, et al. R-2HG Exhibits Anti-tumor Activity by Targeting FTO/m(6)A/MYC/CEBPA Signaling. Cell. 2018;172(1-2):90-105. e123.

46. Yang S, Wei J, Cui YH, Park G, Shah P, Deng Y, Aplin AE, Lu Z, Hwang S, He C, He YY. m(6)A mRNA demethylase FTO regulates melanoma tumorigenicity and response to anti-PD-1 blockade. Nat Commun. 2019;10(1):2782.

Figures

A

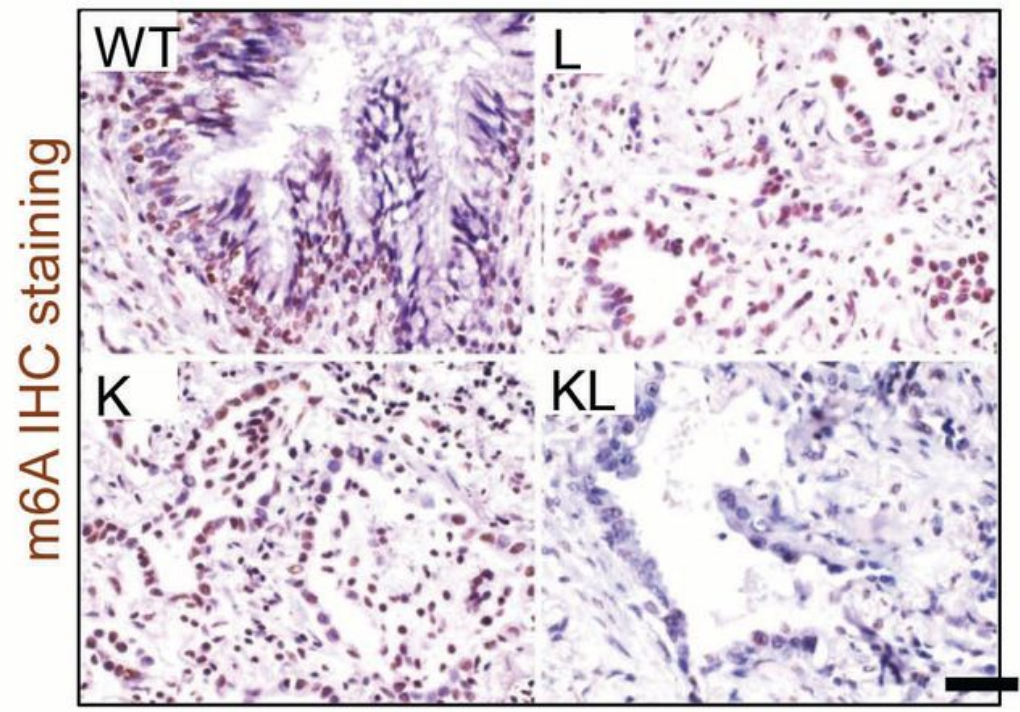

C

\begin{tabular}{|l|c|c|}
\hline Characters & $\begin{array}{c}\text { m6A, } r \\
(\text { KRASWT })\end{array}$ & $\begin{array}{c}\text { m6A, } r \\
\left(\text { Kras }^{\text {Mut }}\right)\end{array}$ \\
\hline Age & 0.109 & -0.153 \\
\hline Smoking & 0.228 & -0.047 \\
\hline Tumor size & -0.030 & $-0.307^{*}$ \\
\hline Differentiation & 0.218 & $0.331^{*}$ \\
\hline TNM stage & 0.084 & $-0.381^{*}$ \\
\hline Clinical stage & 0.095 & $-0.332^{*}$ \\
\hline
\end{tabular}

B

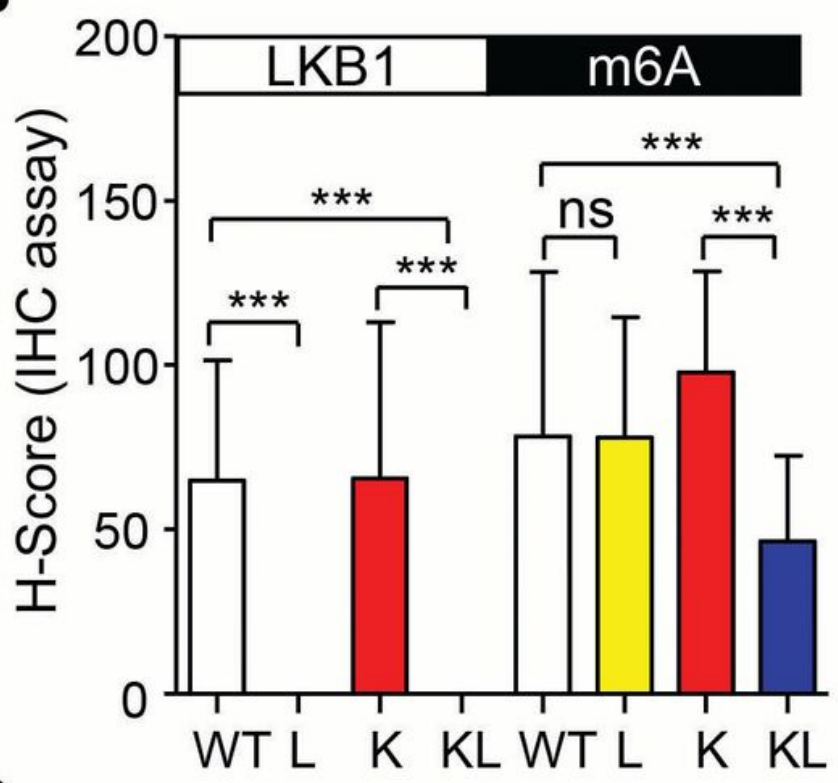

D

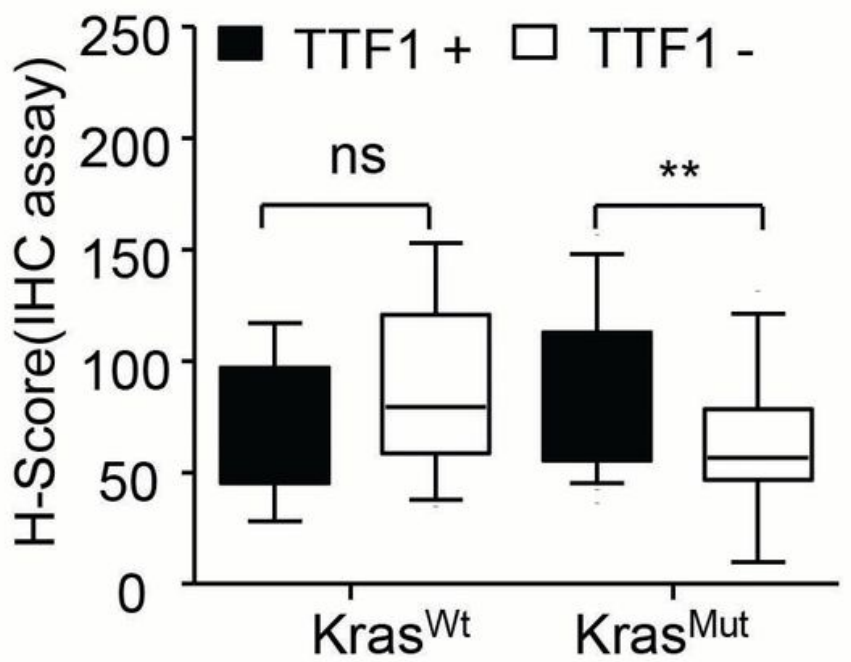

Figure 1 
Reduced m6A modification is associated with the aggressiveness of lung cancer with KRAS and LKB1 mutation. (A, B) Representation and quantification of LKB1 expression and m6A level in lung cancer patients. Data are mean \pm SD. Bar $=50 \mu \mathrm{m}$. (C) Spearman correlation analysis of m6A level with the clinical characters of KRAS mutant or wildtype lung cancer patients. (D) The m6A level in lung cancer patients with TTF-1 positive or negative. Boxes and whiskers represent the 10th to 90th percentiles, respectively; the median is the central line in each box. ${ }^{*} \mathrm{P}<0.01,{ }^{\star *} \mathrm{P}<0.001$ by Student's $\mathrm{t}$ test. KRAS Mut; LKB1 Mut (KL); KRAS Mut; LKB1 Wt (K); KRAS Wt; LKB1 Mut (L) and KRAS Wt; LKB1 Wt (WT).
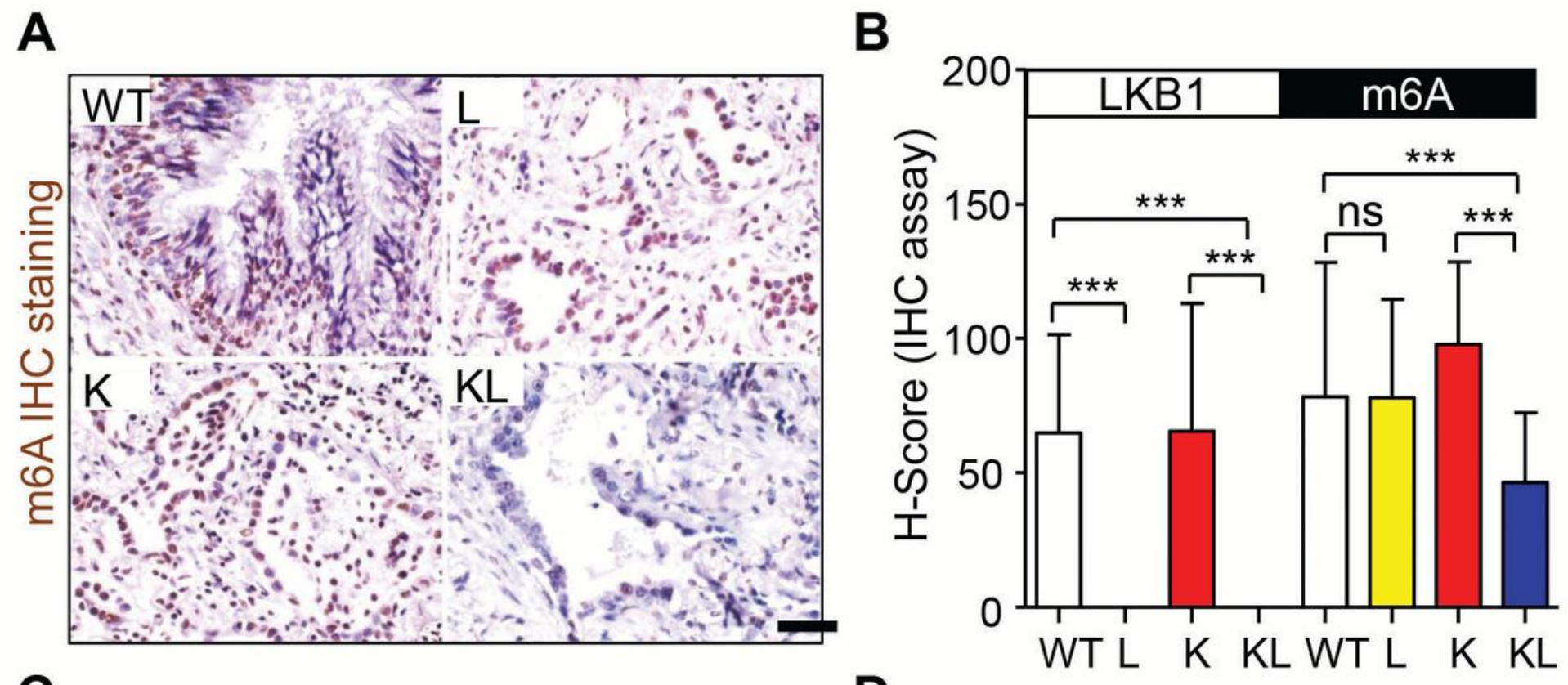

\section{C}

\begin{tabular}{|l|c|c|}
\hline Characters & $\begin{array}{c}\text { m6A, } r \\
(\text { KRASWT })\end{array}$ & $\begin{array}{c}\text { m6A, r } \\
\left(K^{\text {Kas }}{ }^{\text {Mut }}\right)\end{array}$ \\
\hline Age & 0.109 & -0.153 \\
\hline Smoking & 0.228 & -0.047 \\
\hline Tumor size & -0.030 & $-0.307^{*}$ \\
\hline Differentiation & 0.218 & $0.331^{*}$ \\
\hline TNM stage & 0.084 & $-0.381^{*}$ \\
\hline Clinical stage & 0.095 & $-0.332^{*}$ \\
\hline
\end{tabular}

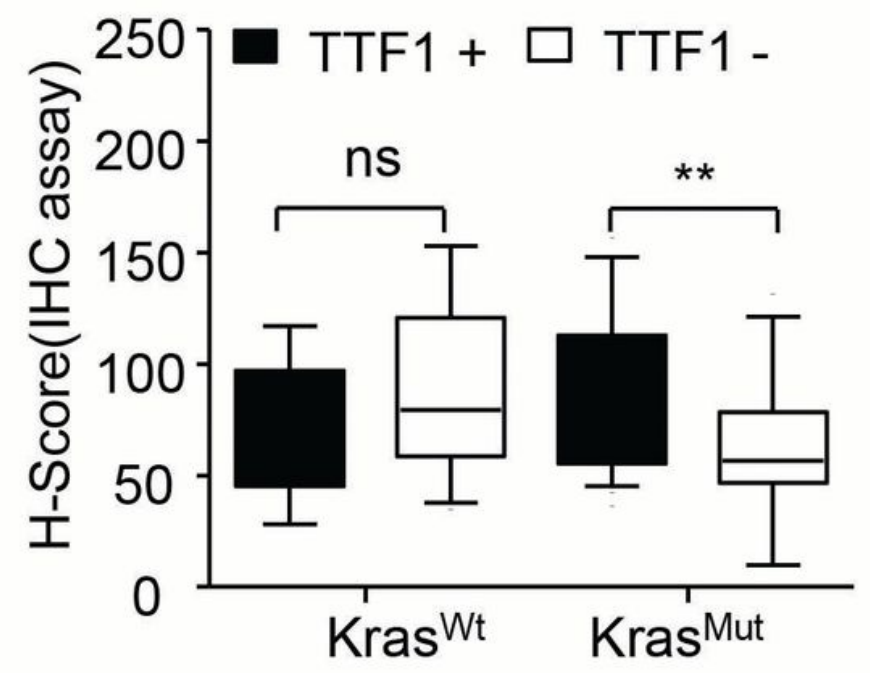

Figure 1

Reduced m6A modification is associated with the aggressiveness of lung cancer with KRAS and LKB1 mutation. (A, B) Representation and quantification of LKB1 expression and m6A level in lung cancer patients. Data are mean \pm SD. Bar $=50 \mu \mathrm{m}$. (C) Spearman correlation analysis of m6A level with the clinical characters of KRAS mutant or wildtype lung cancer patients. (D) The m6A level in lung cancer 
patients with TTF-1 positive or negative. Boxes and whiskers represent the 10th to 90th percentiles, respectively; the median is the central line in each box. ${ }^{\star \star} \mathrm{P}<0.01,{ }^{*} * \mathrm{P}<0.001$ by Student's t test. KRAS Mut; LKB1 Mut (KL); KRAS Mut; LKB1 Wt (K); KRAS Wt; LKB1 Mut (L) and KRAS Wt; LKB1 Wt (WT).
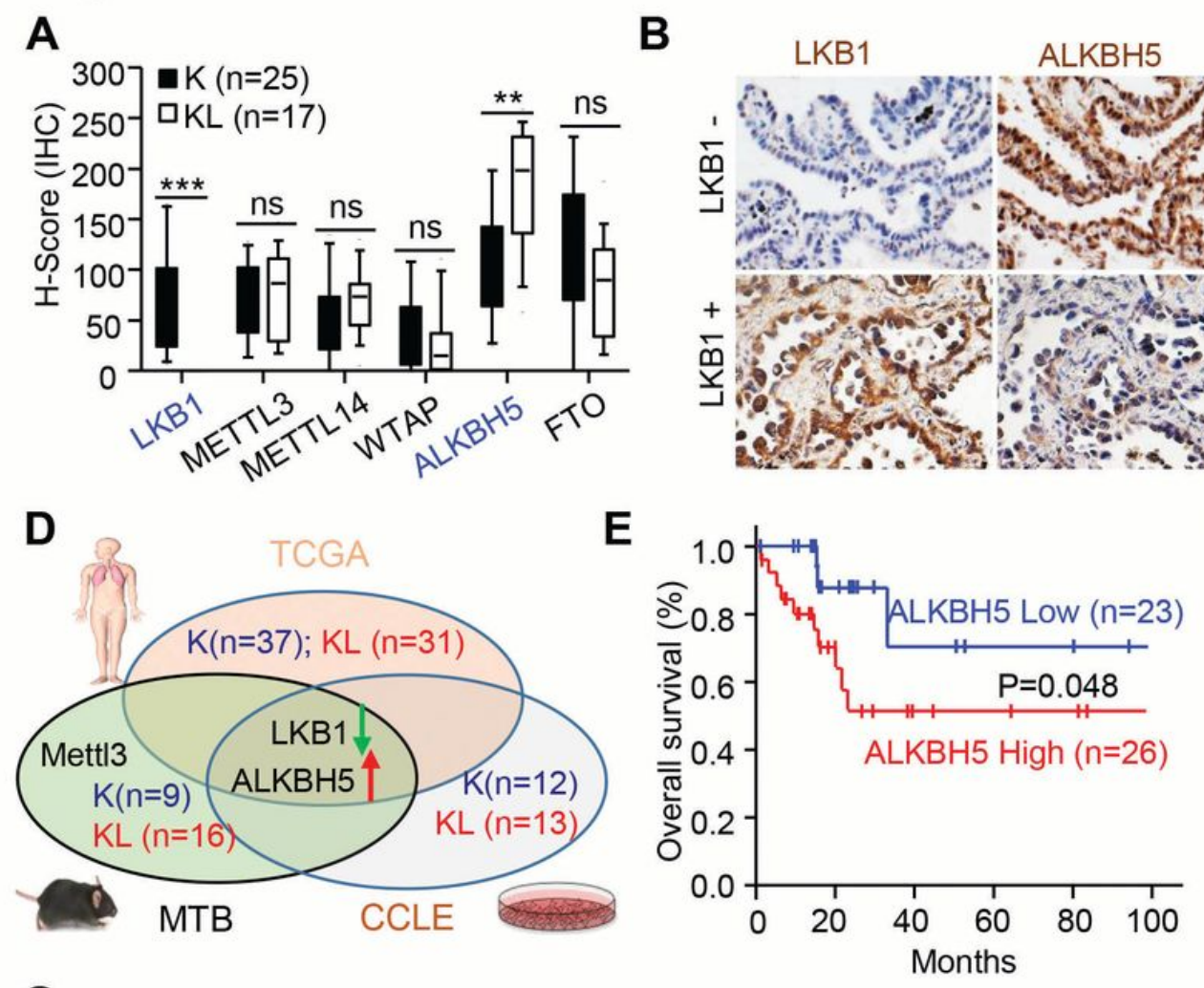

C

\begin{tabular}{|l|c|}
\hline Characters & ALKBH5, $r$ \\
\hline m6A level & $-0.326^{*}$ \\
\hline Age & -0.011 \\
\hline Smoking & 0.003 \\
\hline Tumor size & $0.325^{*}$ \\
\hline Differentiation & -0.194 \\
\hline TNM stage & $0.418^{\star *}$ \\
\hline Clinical stage & $0.333^{*}$ \\
\hline
\end{tabular}

$\mathbf{F}$

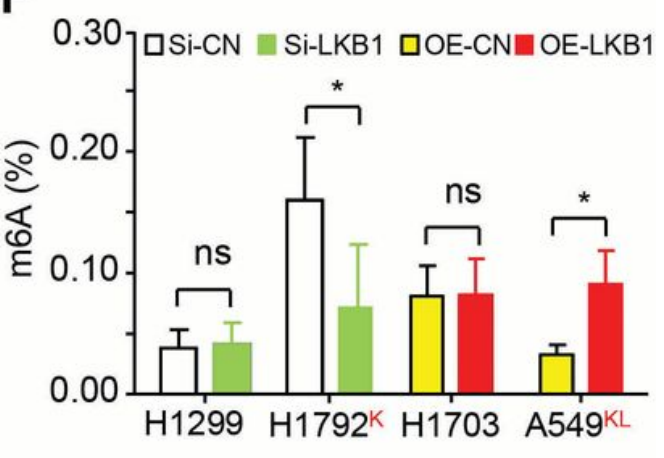

G
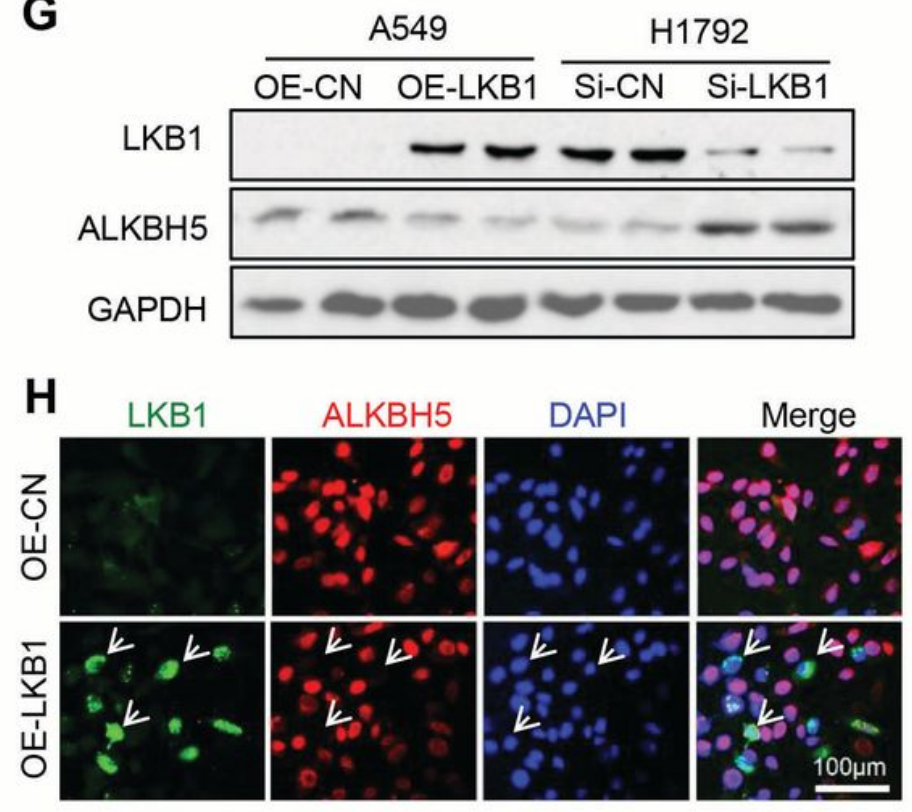
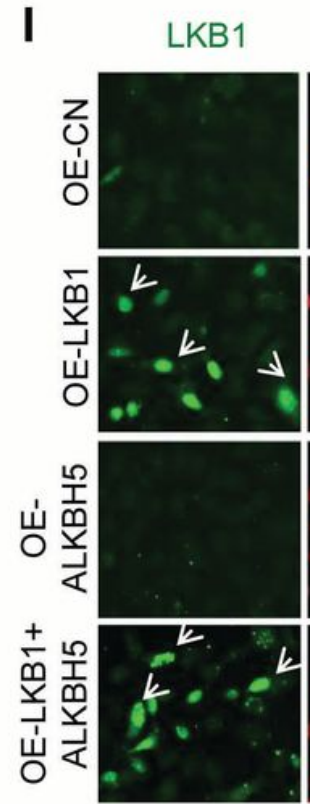

m6A
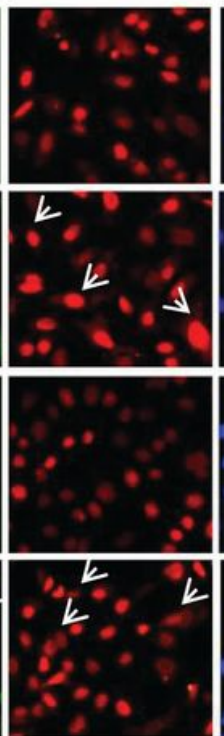

DAPI
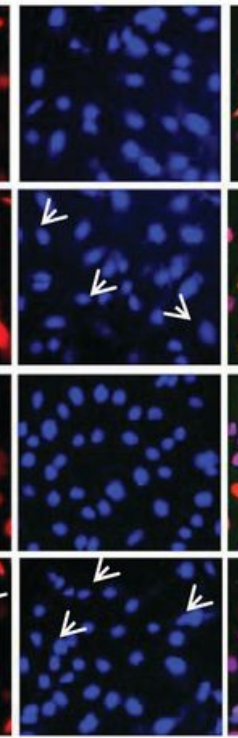

Merge

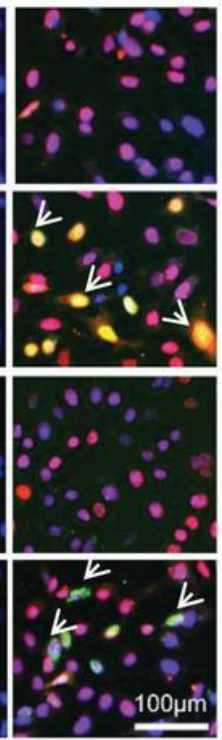

Figure 2

LKB1 loss upregulated ALKBH5 responses to m6A reduction in KRAS mutant lung cancer. (A, B) Quantification and representation of $L K B 1$ and $m 6 \mathrm{~A}$ modulators in $\mathrm{KL}$ relative to $\mathrm{K}$ lung cancer patients. Boxes and whiskers represent the 10th to 90th percentiles, respectively; the median is the central line in each box. Bar=50 $\mu \mathrm{m}$. (C) Spearman correlation analysis of ALKBH5 expression with the lung cancer 
pathological characters. (D) Venn diagram showing the differentially expressed genes (DEGs) of LKB1, m6A modulators and readers between $\mathrm{KL}$ and $\mathrm{K}$ lung cancer. (E) Kaplan-Meier survival curve of patients with high and low ALKBH5 mRNA expression from the TCGA dataset. (F) Global m6A level regulation by LKB1 in lung cancer cell lines. $(G)$ Western blot showing the LKB1 and ALKBH5 protein expression. $(H)$ Co-immunofluorescence staining for LKB1 and ALKBH5 in A549 cells. Arrows show LKB1 positive and ALKBH5 negative cells. (I) LKB1 and m6A co-staining by LKB1 and/or ALKBH5 over-expression in A549 cells. Arrows show cells of LKB1 positive and m6A with strong (Second panel) or weak (Forth panel) intensity. Data are mean $\pm S D$ in $F(n=3)$. ${ }^{*} P<0.05$ (Student's $t$ test). Si-CN, Si-RNA-A; OE-CN, OE-C-Flag pcDNA3; OE, over-expression. KRAS Mut; LKB1 Mut (KL); KRAS Mut; LKB1 Wt (K).
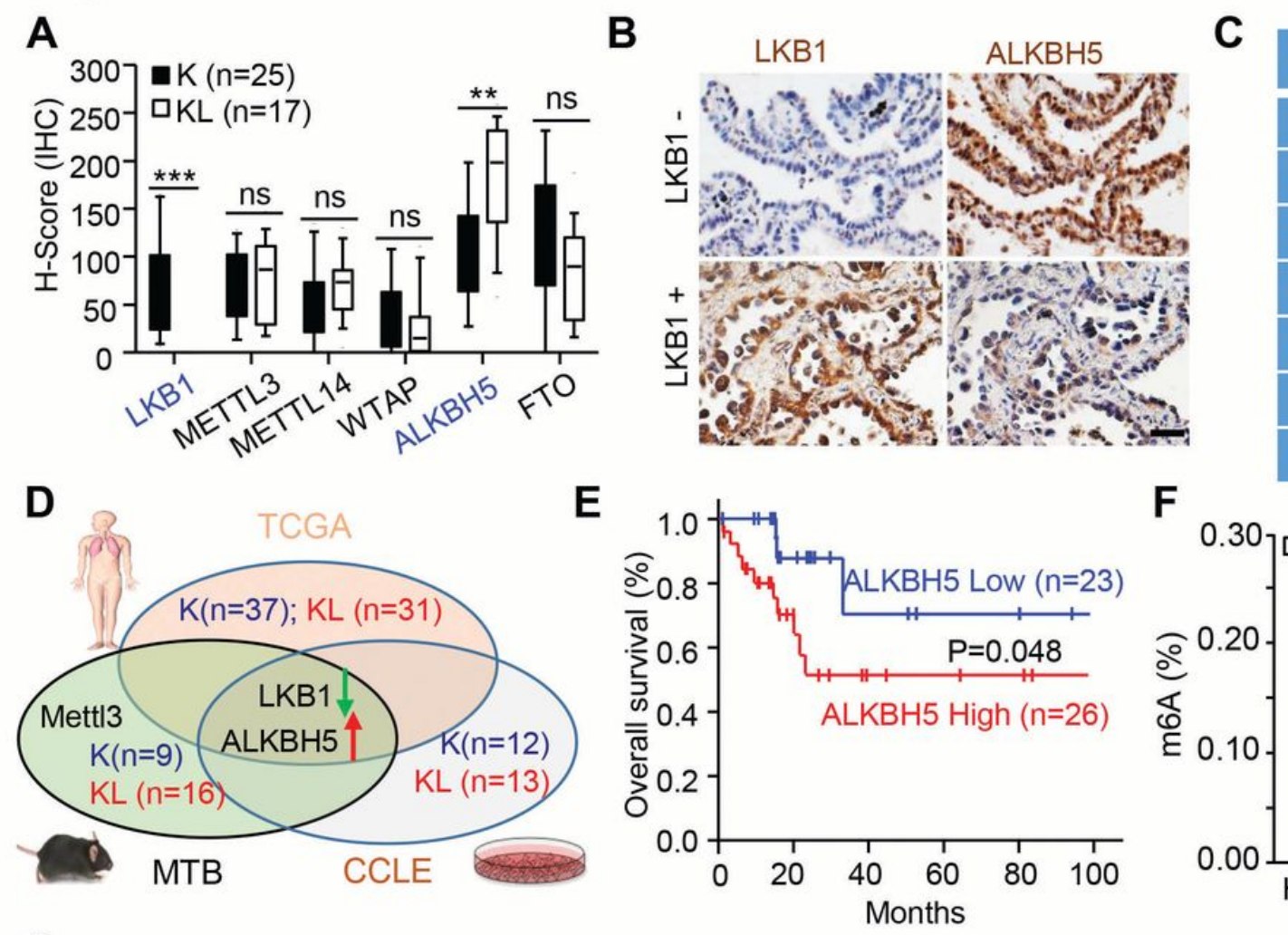

\begin{tabular}{|l|c|}
\hline Characters & ALKBH5, $r$ \\
\hline m6A level & $-0.326^{*}$ \\
\hline Age & -0.011 \\
\hline Smoking & 0.003 \\
\hline Tumor size & $0.325^{\star}$ \\
\hline Differentiation & -0.194 \\
\hline TNM stage & $0.418^{* *}$ \\
\hline Clinical stage & $0.333^{*}$ \\
\hline
\end{tabular}

$\mathbf{F}$

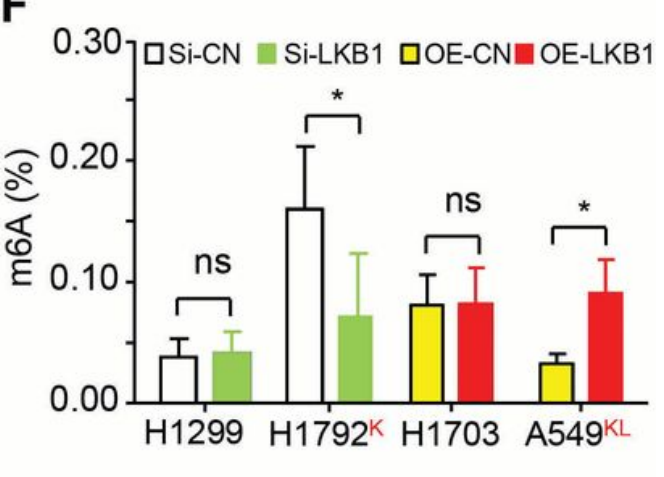

G
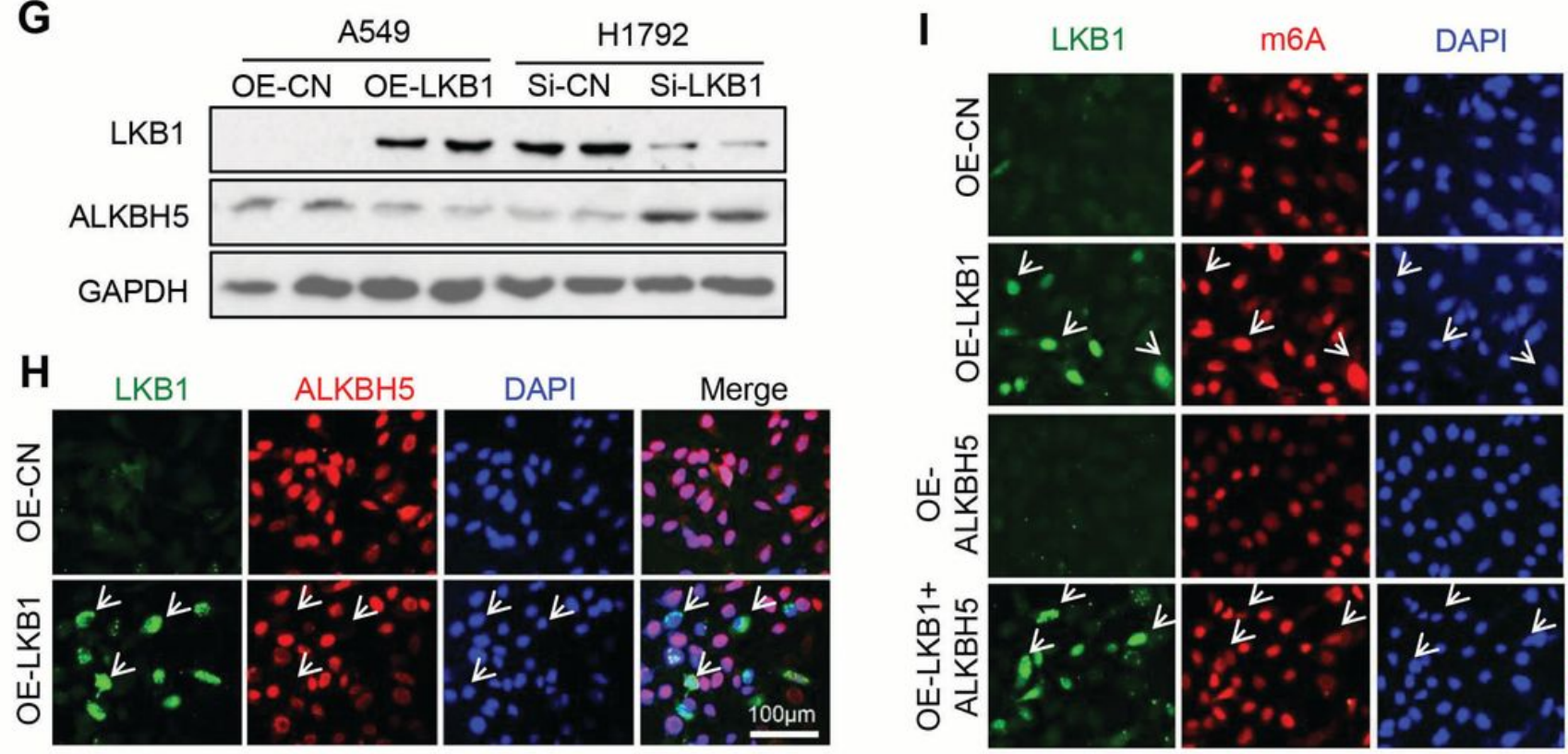

Merge

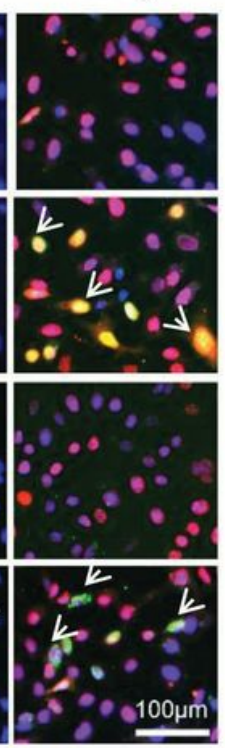




\section{Figure 2}

LKB1 loss upregulated ALKBH5 responses to m6A reduction in KRAS mutant lung cancer. (A, B) Quantification and representation of LKB1 and m6A modulators in KL relative to K lung cancer patients. Boxes and whiskers represent the 10th to 90th percentiles, respectively; the median is the central line in each box. Bar=50 $\mu \mathrm{m}$. (C) Spearman correlation analysis of ALKBH5 expression with the lung cancer pathological characters. (D) Venn diagram showing the differentially expressed genes (DEGs) of LKB1, m6A modulators and readers between $\mathrm{KL}$ and $\mathrm{K}$ lung cancer. (E) Kaplan-Meier survival curve of patients with high and low ALKBH5 mRNA expression from the TCGA dataset. (F) Global m6A level regulation by LKB1 in lung cancer cell lines. $(G)$ Western blot showing the LKB1 and ALKBH5 protein expression. $(H)$ Co-immunofluorescence staining for LKB1 and ALKBH5 in A549 cells. Arrows show LKB1 positive and ALKBH5 negative cells. (I) LKB1 and m6A co-staining by LKB1 and/or ALKBH5 over-expression in A549 cells. Arrows show cells of LKB1 positive and m6A with strong (Second panel) or weak (Forth panel) intensity. Data are mean $\pm S D$ in $F(n=3)$. ${ }^{*} P<0.05$ (Student's t test). Si-CN, Si-RNA-A; OE-CN, OE-c-Flag pcDNA3; OE, over-expression. KRAS Mut; LKB1 Mut (KL); KRAS Mut; LKB1 Wt (K). 
A

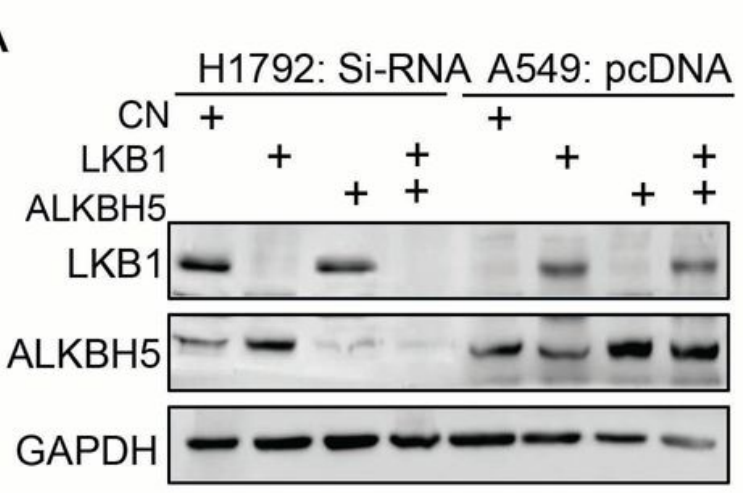

D
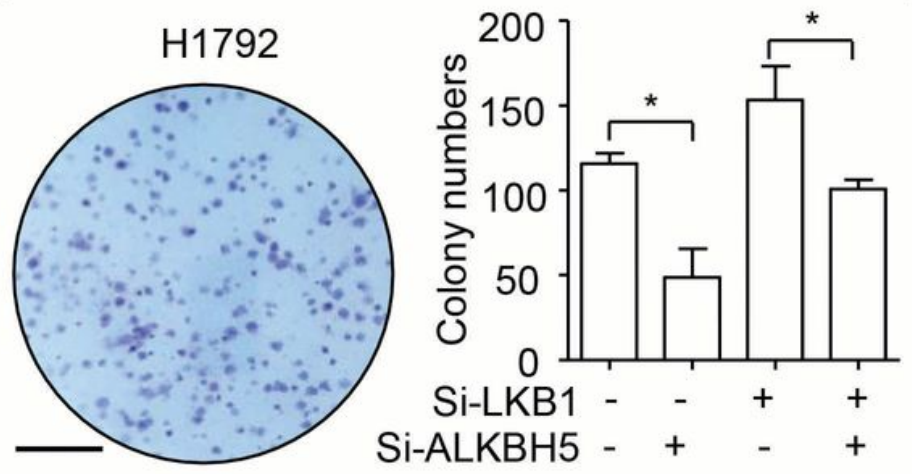

$E$

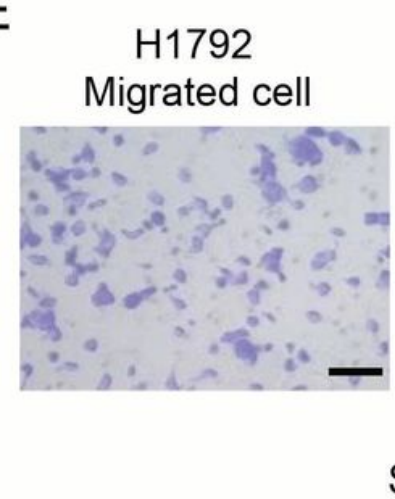

C
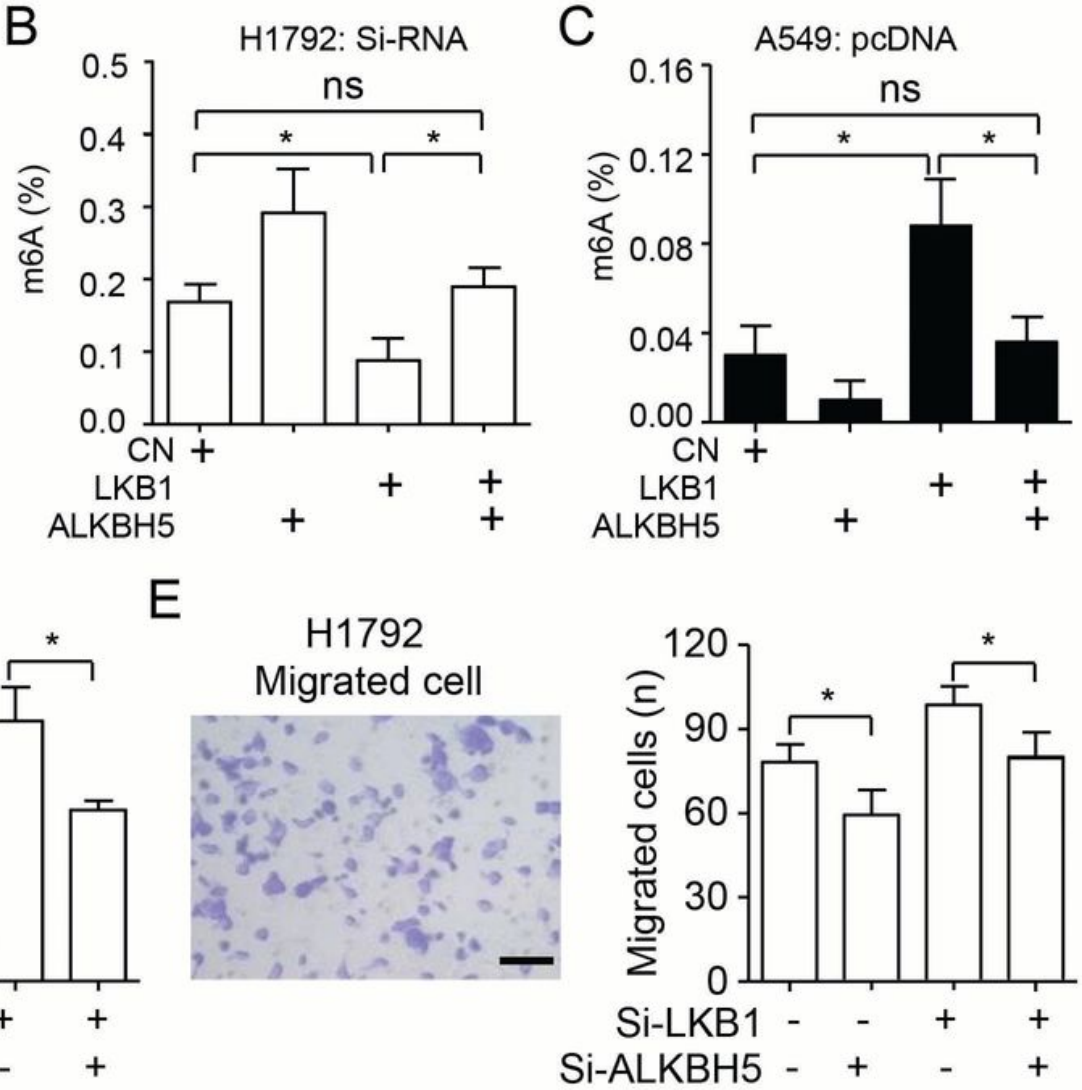

$\mathrm{F}$

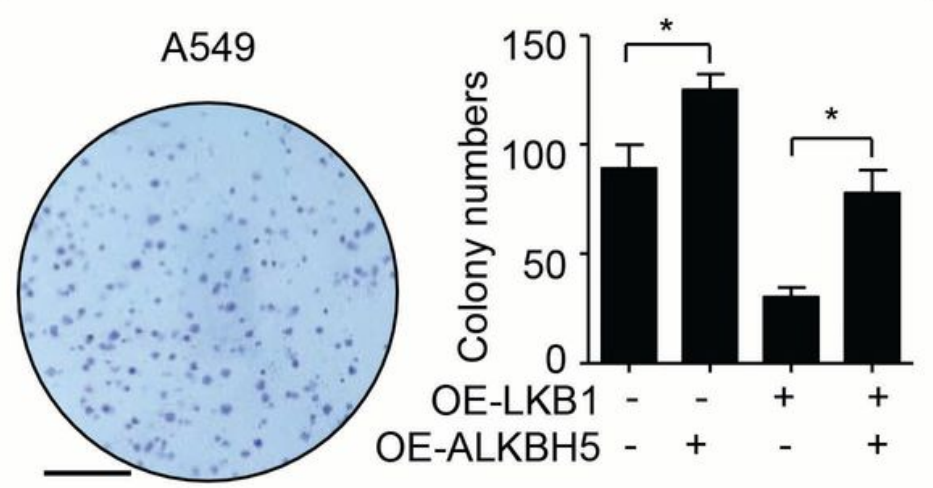

G

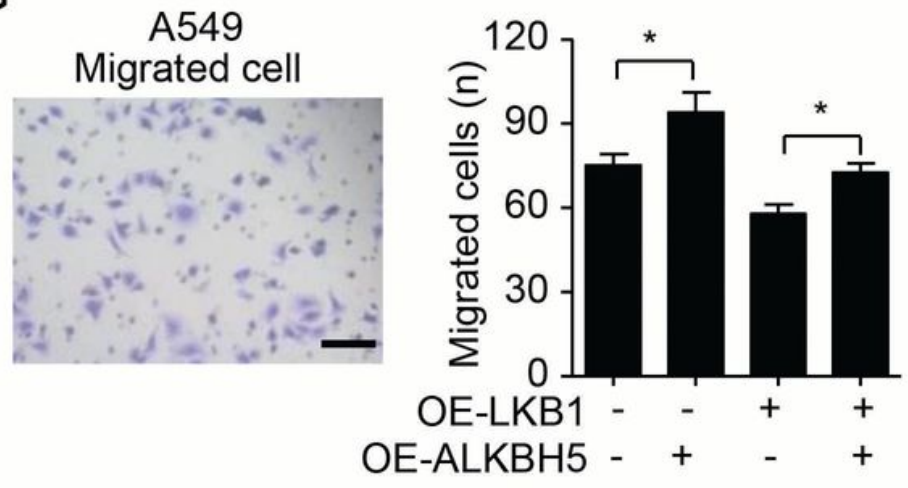

Figure 3

ALKBH5 increases the lung cancer cell proliferation and migration. (A) Western blot analysis shown that LKB1 and ALKBH5 were successfully knocked down in H1792 cells and over-expressed in A549 cells. (B, C) the measurement of global m6A by ELISA assay in H1792 (B) or A549 (C) cells, respectively. The colony formation ability (D) and cell migration (E) were reduced at both of basal level and LKB1 silenced level by ALKBH5 knock down in $\mathrm{H} 1792$ cells, respectively. The reversible effect was found at both of basal level and LKB1 over-expressed level by ALKBH5 transfected in A549 cells using colony formation $(F)$ and transwell assay $(G)$. Data are mean $\pm S D(n=3)$ and were analyzed by one-way ANOVA, followed by Bonferroni's multiple comparison test for B-G. ${ }^{*} \mathrm{P}<0.05$. Bar=5 mm in $\mathrm{D}$ and $\mathrm{F}$; Bar=50 $\mu \mathrm{m}$ in $\mathrm{E}$ and $\mathrm{G}$. 
A

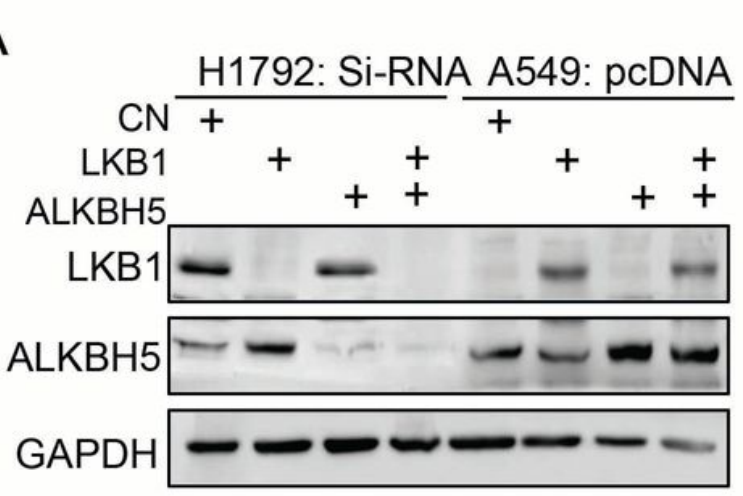

$B$
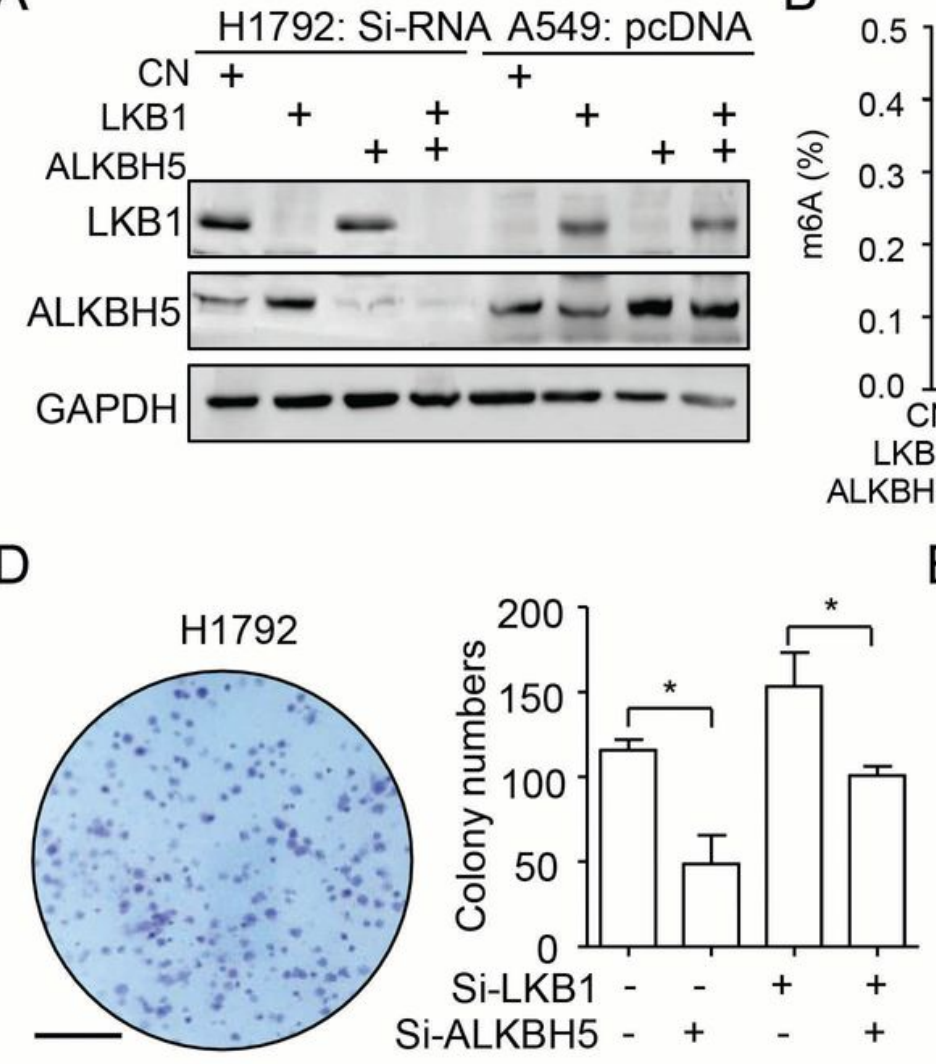

E

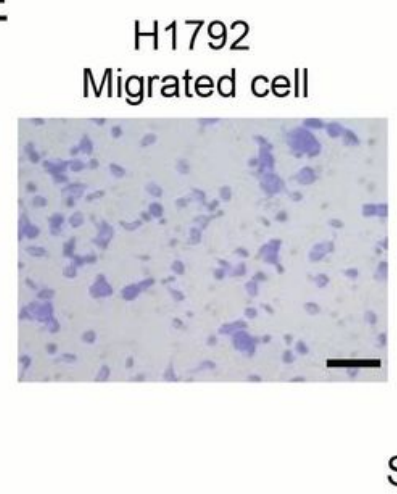

C

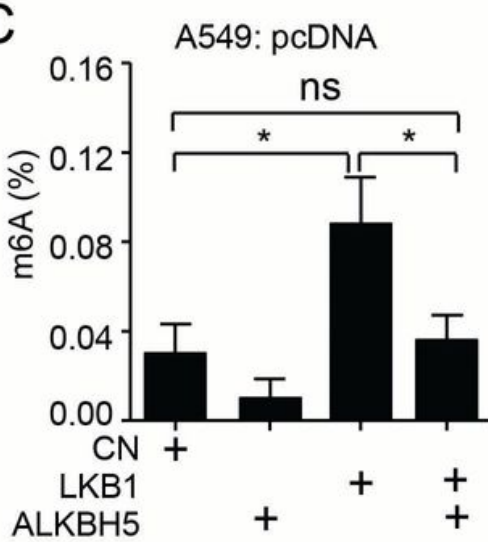

$\mathrm{F}$

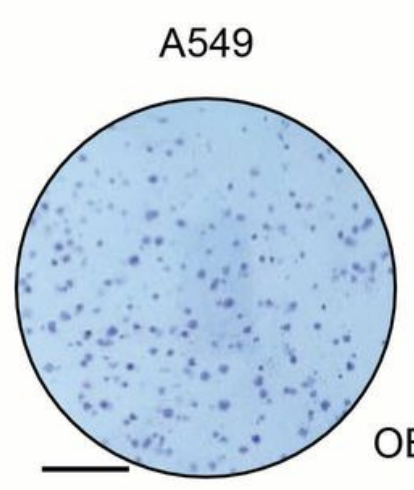

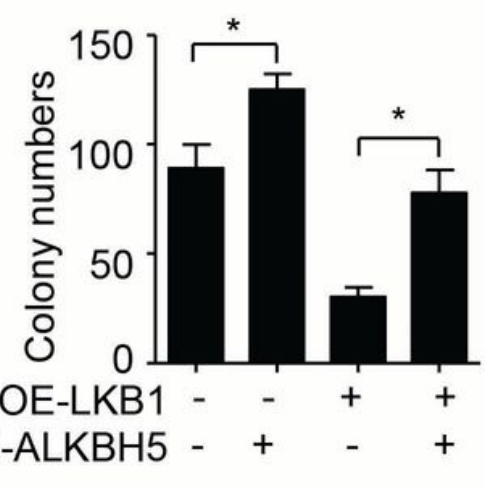

G

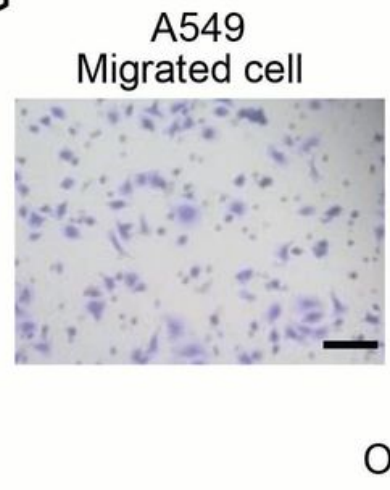

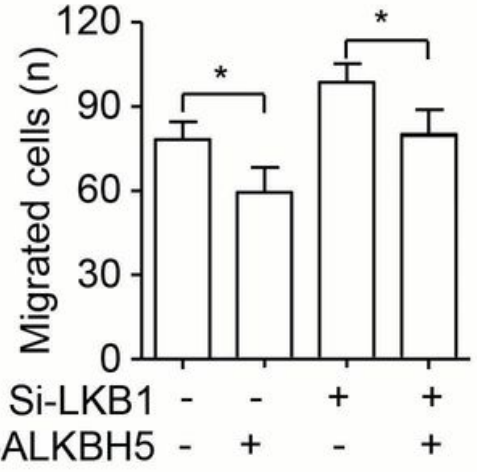

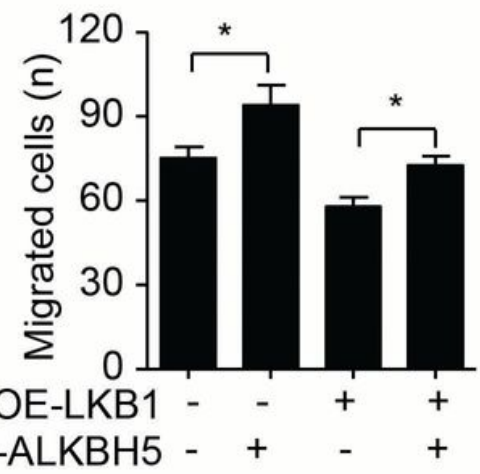

Figure 3

ALKBH5 increases the lung cancer cell proliferation and migration. (A) Western blot analysis shown that LKB1 and ALKBH5 were successfully knocked down in H1792 cells and over-expressed in A549 cells. (B, C) the measurement of global m6A by ELISA assay in H1792 (B) or A549 (C) cells, respectively. The colony formation ability (D) and cell migration (E) were reduced at both of basal level and LKB1 silenced level by ALKBH5 knock down in $\mathrm{H} 1792$ cells, respectively. The reversible effect was found at both of basal level and LKB1 over-expressed level by ALKBH5 transfected in A549 cells using colony formation $(F)$ and transwell assay $(G)$. Data are mean $\pm S D(n=3)$ and were analyzed by one-way ANOVA, followed by Bonferroni's multiple comparison test for B-G. ${ }^{*} \mathrm{P}<0.05$. Bar=5 mm in $\mathrm{D}$ and $\mathrm{F}$; Bar=50 $\mu \mathrm{m}$ in $\mathrm{E}$ and $\mathrm{G}$. 

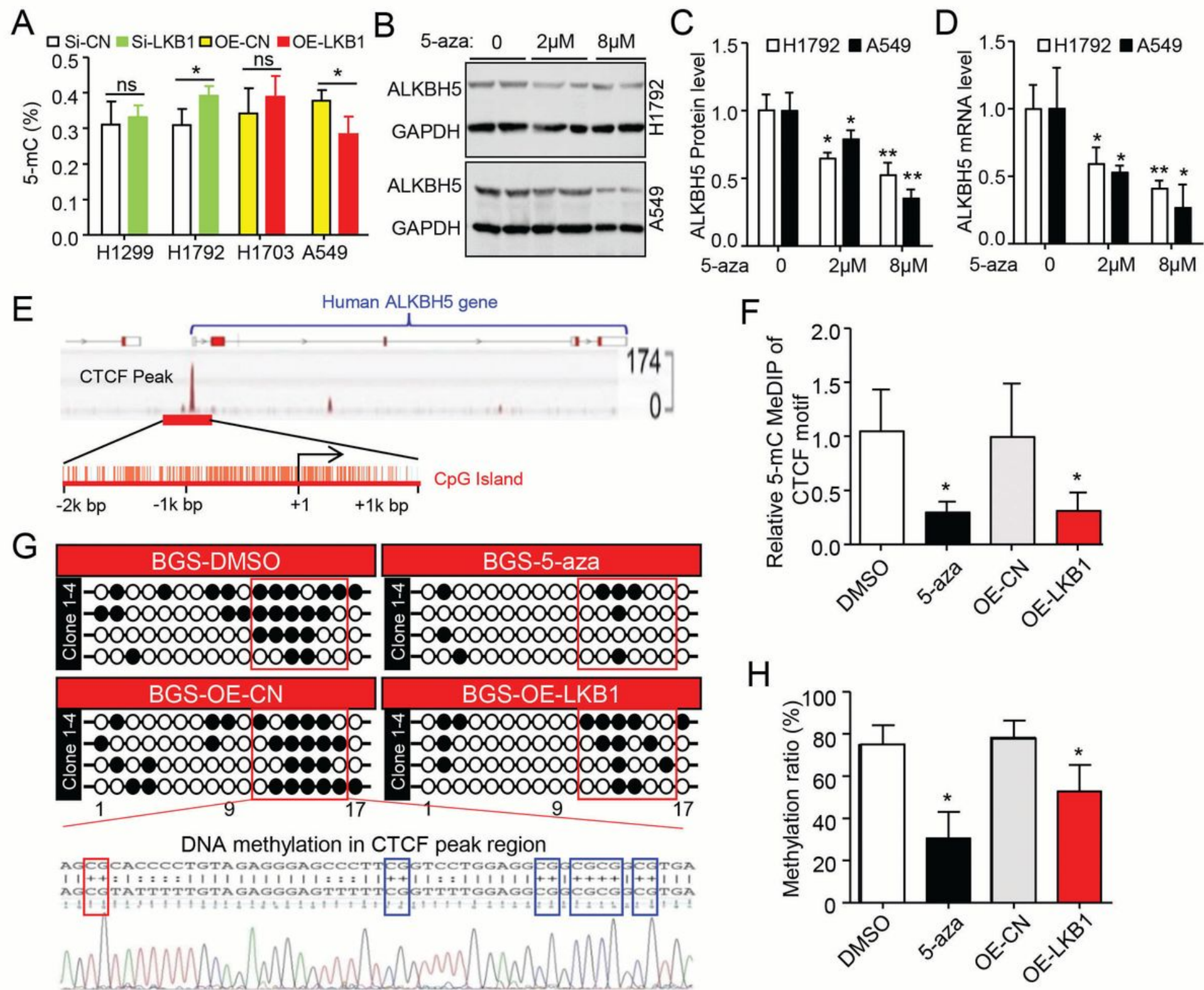

Figure 4

DNA hypermethylation upregulates ALKBH5 in KL lung cancer cells. (A) 5mC DNA methylation was detected by ELISA assay. Data as mean $\pm S D(n=3),{ }^{*} P<0.05$ (Student's t test). (B-D) Representation and quantification of ALKBH5 expression at protein $(B, C)$ and $m R N A$ levels $(D)$ in response to 5-aza treatment in A549 and $\mathrm{H} 1792$ cells for 72 hrs. Error bars, SD ( $n=4$ for WB; $n=3$ for qRT-PCR), * $P<0.05$ as compared their corresponding controls (2-way ANOVA with Bonferroni multiple comparison post hoc test). (E) CTCF peak located in the CpGs Island of human ALKBH5 gene promoter. Bottom panel: the Methprimer histogram of $\mathrm{CpG}$ islands ( $\mathrm{CpGI}$, red box) and $\mathrm{CpG}$ dinucleotides (red vertical lines) in the regulatory region of ALKBH5. (F) MeDIP assay showing the decrease of $5 \mathrm{mC}$ enrichment on the ALKBH5 promoter containing CTCF motif fragment by treated with 5-aza and LKB1 over-expression. (G, H) Representative bisulfite sequencing of 4 clones $(\mathrm{G})$ and quantification of DNA methylation $(\mathrm{H})$ of ALKBH5 promoter 
containing CTCF motif. $\mathrm{n}=4$ in $\mathrm{F}$ and $\mathrm{H}$, mean $\pm \mathrm{SD}$, * $\mathrm{P}<0.05$ vs. A549 DMSO by one-way ANOVA followed by Tukey's test in F and H. Si-CN, Si-RNA-A. OE-CN, OE-c-Flag pcDNA3. OE, over-expression.
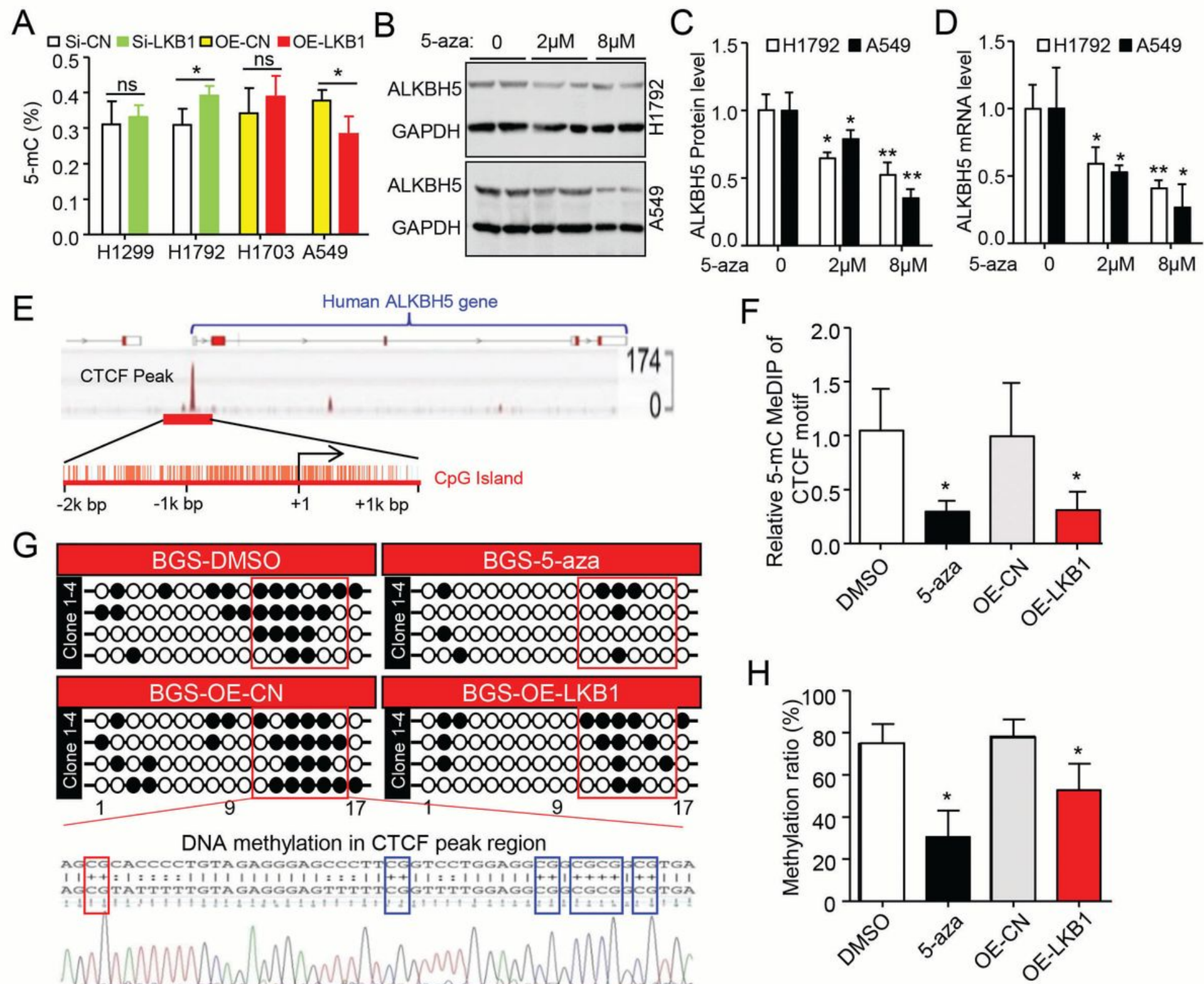

Figure 4

DNA hypermethylation upregulates ALKBH5 in KL lung cancer cells. (A) 5mC DNA methylation was detected by ELISA assay. Data as mean $\pm S D(n=3)$, *P $<0.05$ (Student's t test). (B-D) Representation and quantification of ALKBH5 expression at protein $(B, C)$ and mRNA levels $(D)$ in response to 5-aza treatment in A549 and H1792 cells for 72 hrs. Error bars, SD ( $=4$ for WB; $n=3$ for qRT-PCR), *P < 0.05 as compared their corresponding controls (2-way ANOVA with Bonferroni multiple comparison post hoc test). (E) CTCF peak located in the CpGs Island of human ALKBH5 gene promoter. Bottom panel: the Methprimer histogram of $\mathrm{CpG}$ islands (CpGI, red box) and $\mathrm{CpG}$ dinucleotides (red vertical lines) in the regulatory region of $\mathrm{ALKBH5}$ (F) MeDIP assay showing the decrease of $5 \mathrm{mC}$ enrichment on the ALKBH5 promoter containing CTCF motif fragment by treated with 5-aza and LKB1 over-expression. (G, H) Representative 
bisulfite sequencing of 4 clones $(\mathrm{G})$ and quantification of DNA methylation $(\mathrm{H})$ of ALKBH5 promoter containing CTCF motif. $\mathrm{n}=4$ in $\mathrm{F}$ and $\mathrm{H}$, mean $\pm \mathrm{SD}$, ${ }^{\mathrm{P}}<0.05$ vs. A549 DMSO by one-way ANOVA followed by Tukey's test in F and H. Si-CN, Si-RNA-A. OE-CN, OE-c-Flag pcDNA3. OE, over-expression.

A

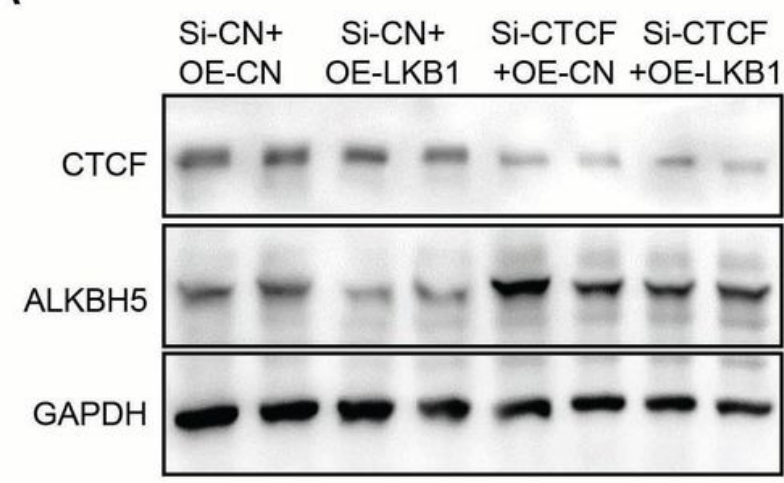

B

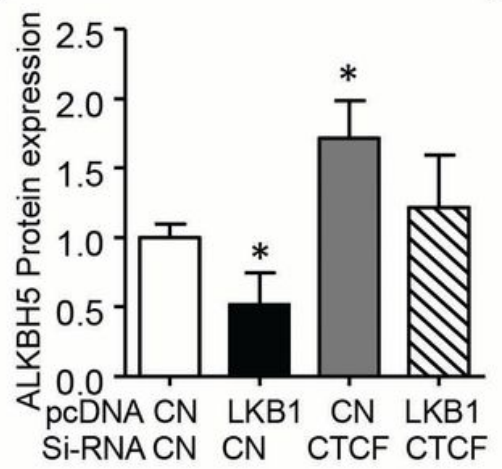

C

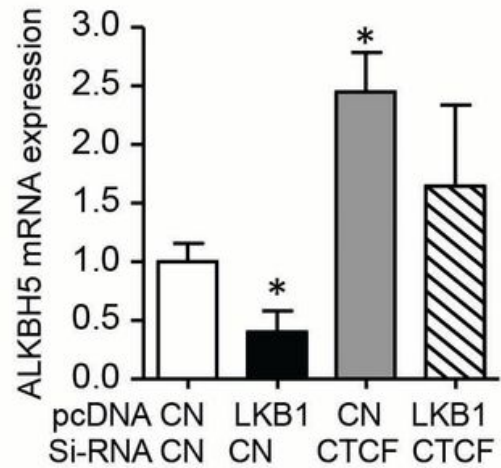

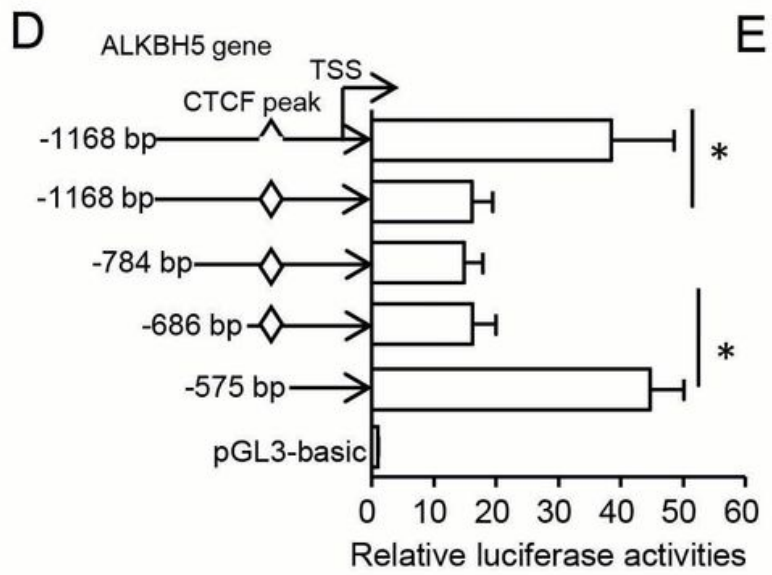

E
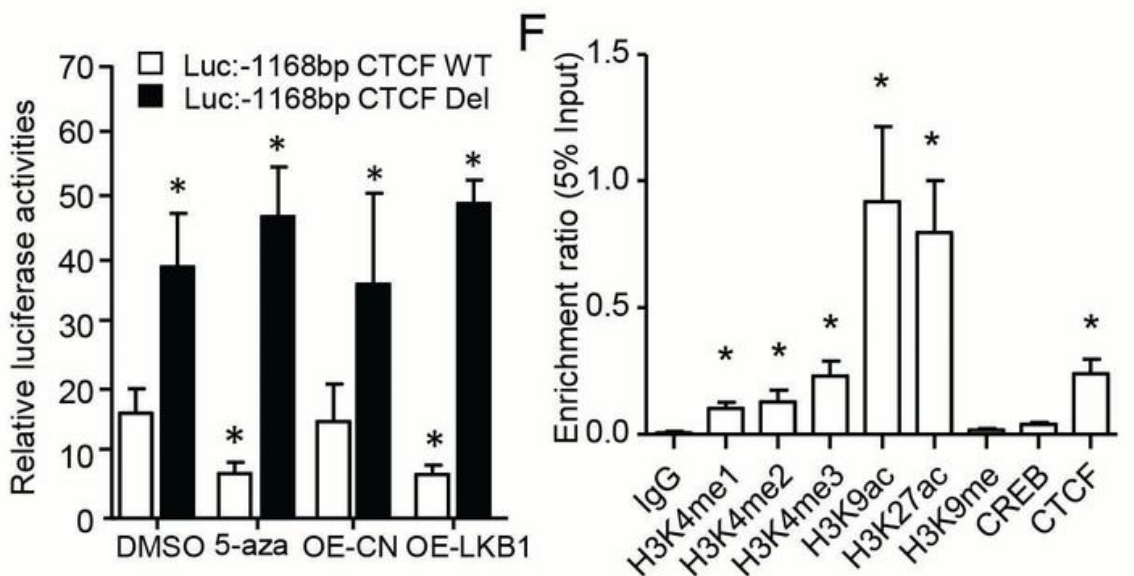

G

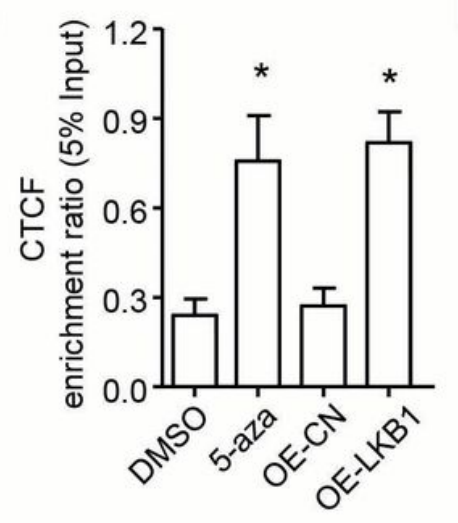

$\mathrm{H}$

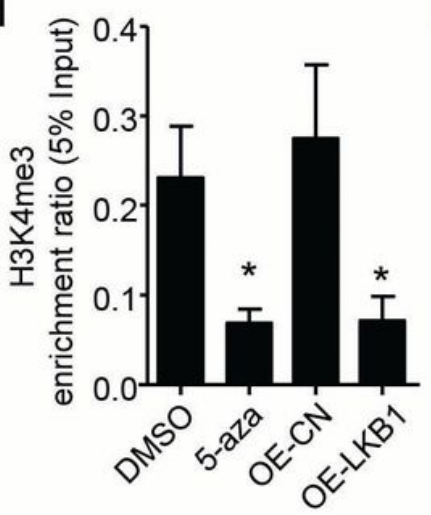

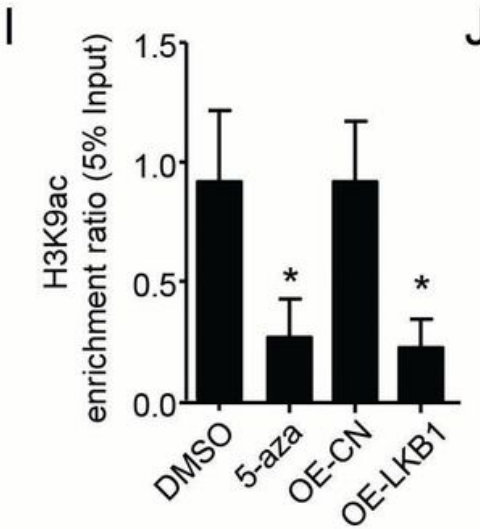

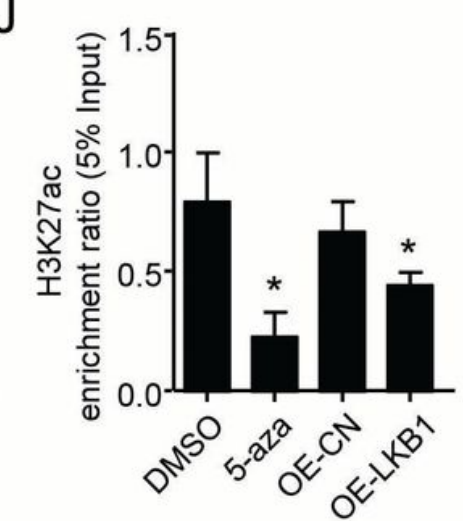

Figure 5

Suppressor-CTCF is required for ALKBH5 down-regulation by LKB1. (A-C) Representative images and quantification of CTCF and ALKBH5 protein expression by WB and qRT-PCR in A549 cells with LKB1 overexpression and/or CTCF knockdown for 48hrs. Error bars, SD ( $n=4$ for WB; $n=5$ for qRT-PCR), $P<0.05$ vs. SI-CN or OE-CN (2-way ANOVA with Bonferroni multiple comparison post hoc test). (D) Luciferase reporter assay of A549 cells transfected with pGL3-basic constructs containing serial LKBH5 promoters or deletion of CTCF peak fragment. $n=8$ /group, mean $\pm S D$. ${ }^{P}<0.05$ by one-way ANOVA followed by 
Tukey's test. (E) Reduction of ALKBH5 Luc:-1168bp wildtype (WT) construct activities by treatment of 5aza or LKB1 over-expression in A549 cells, but not for ALKBH5 Luc:-1168bp deletion (Del). Data as mean $\pm S D(n=5), * P<0.05$ (Student's $t$ test). (F) ChIP-qPCR showing that the ALKBH5-CTCF peak region was occupied by the suppressor of CTCF and activators of histone modulators in A549 cells. (G-J) ChIPqPCR analyses of 5aza treated or LKB1 over-expressed A549 cells. $n=3 /$ group, mean $\pm S D$. ${ }^{*} P 0.05$ vs. IgG in F, vs. A549-DMSO in G-J by one-way ANOVA followed by Tukey's test.

A

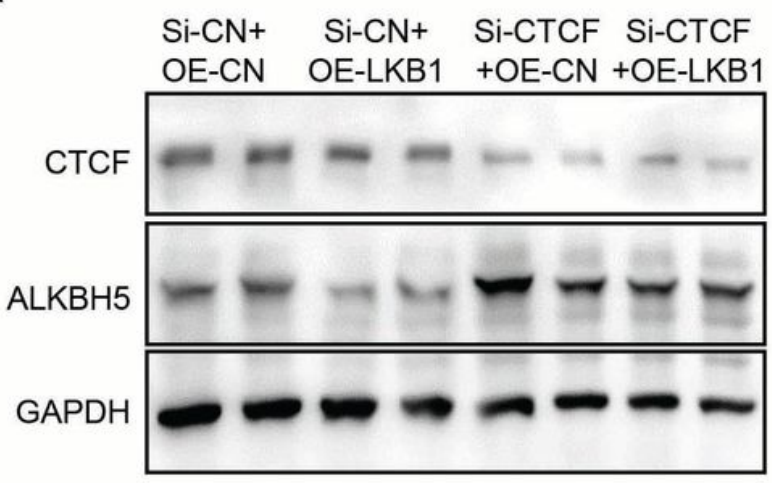

B

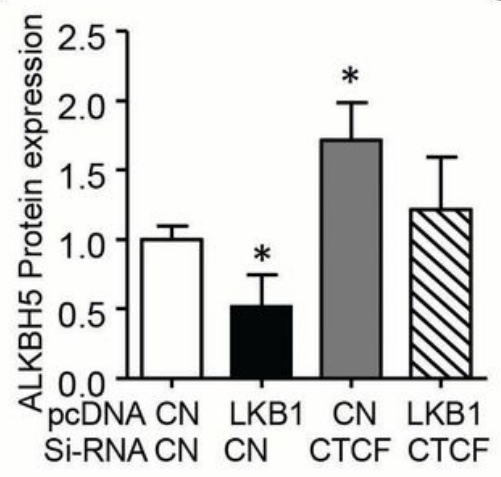

C

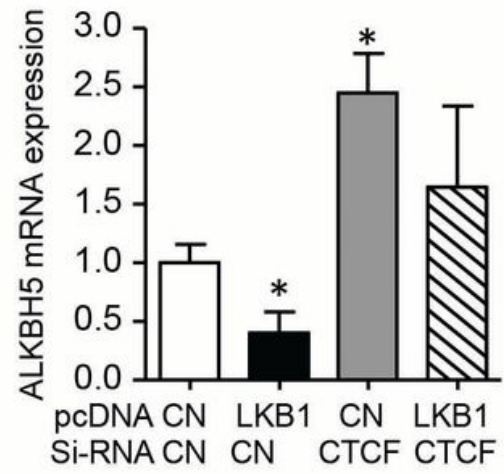

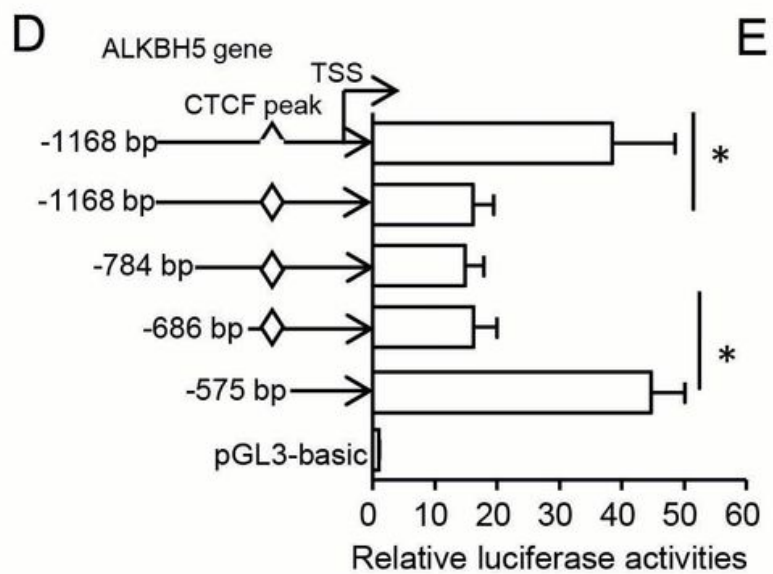
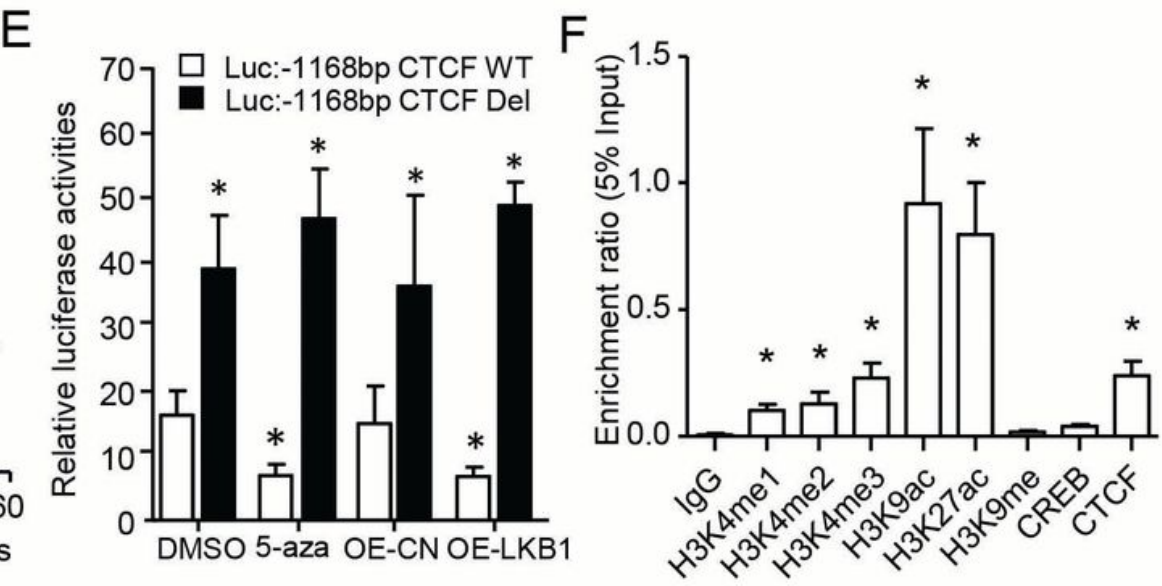

G

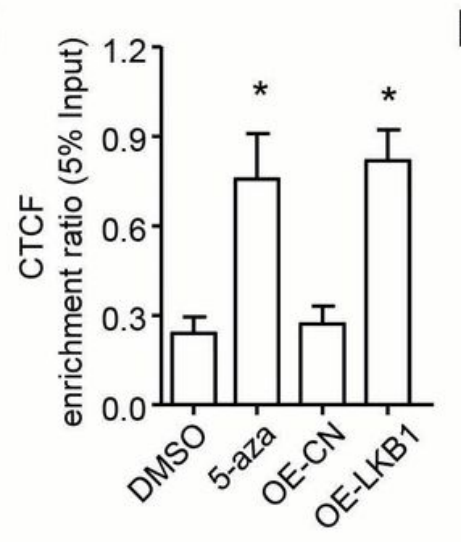

$\mathrm{H}$

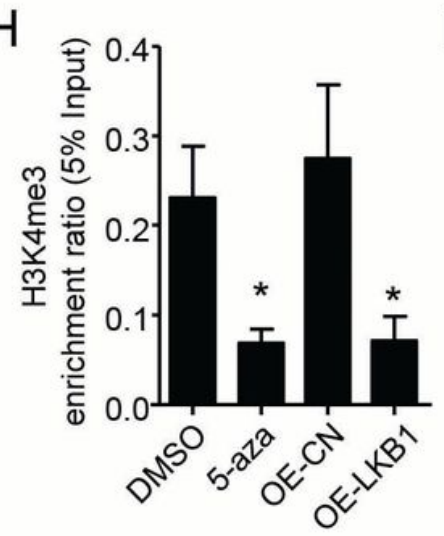

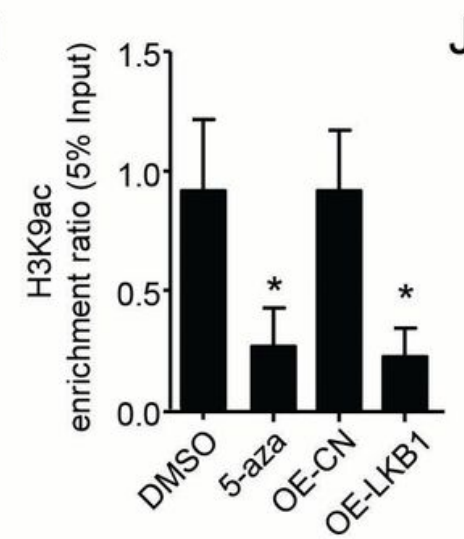

$\mathrm{J}$

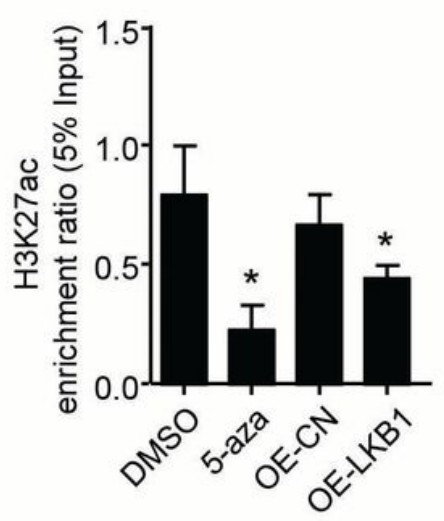

Figure 5

Suppressor-CTCF is required for ALKBH5 down-regulation by LKB1. (A-C) Representative images and quantification of CTCF and ALKBH5 protein expression by WB and qRT-PCR in A549 cells with LKB1 overexpression and/or CTCF knockdown for 48hrs. Error bars, SD ( $n=4$ for WB; $n=5$ for qRT-PCR), *P $<0.05$ 
vs. SI-CN or OE-CN (2-way ANOVA with Bonferroni multiple comparison post hoc test). (D) Luciferase reporter assay of A549 cells transfected with pGL3-basic constructs containing serial LKBH5 promoters or deletion of CTCF peak fragment. $n=8 /$ group, mean $\pm S D$. ${ }^{P} P<0.05$ by one-way ANOVA followed by Tukey's test. (E) Reduction of ALKBH5 Luc:-1168bp wildtype (WT) construct activities by treatment of 5aza or LKB1 over-expression in A549 cells, but not for ALKBH5 Luc:-1168bp deletion (Del). Data as mean $\pm S D(n=5)$, *P $<0.05$ (Student's t test). (F) ChIP-qPCR showing that the ALKBH5-CTCF peak region was occupied by the suppressor of CTCF and activators of histone modulators in A549 cells. (G-J) ChIPqPCR analyses of 5 aza treated or LKB1 over-expressed A549 cells. $n=3$ /group, mean $\pm S D$. ${ }^{*}<<0.05$ vs. IgG in F, vs. A549-DMSO in G-J by one-way ANOVA followed by Tukey's test.
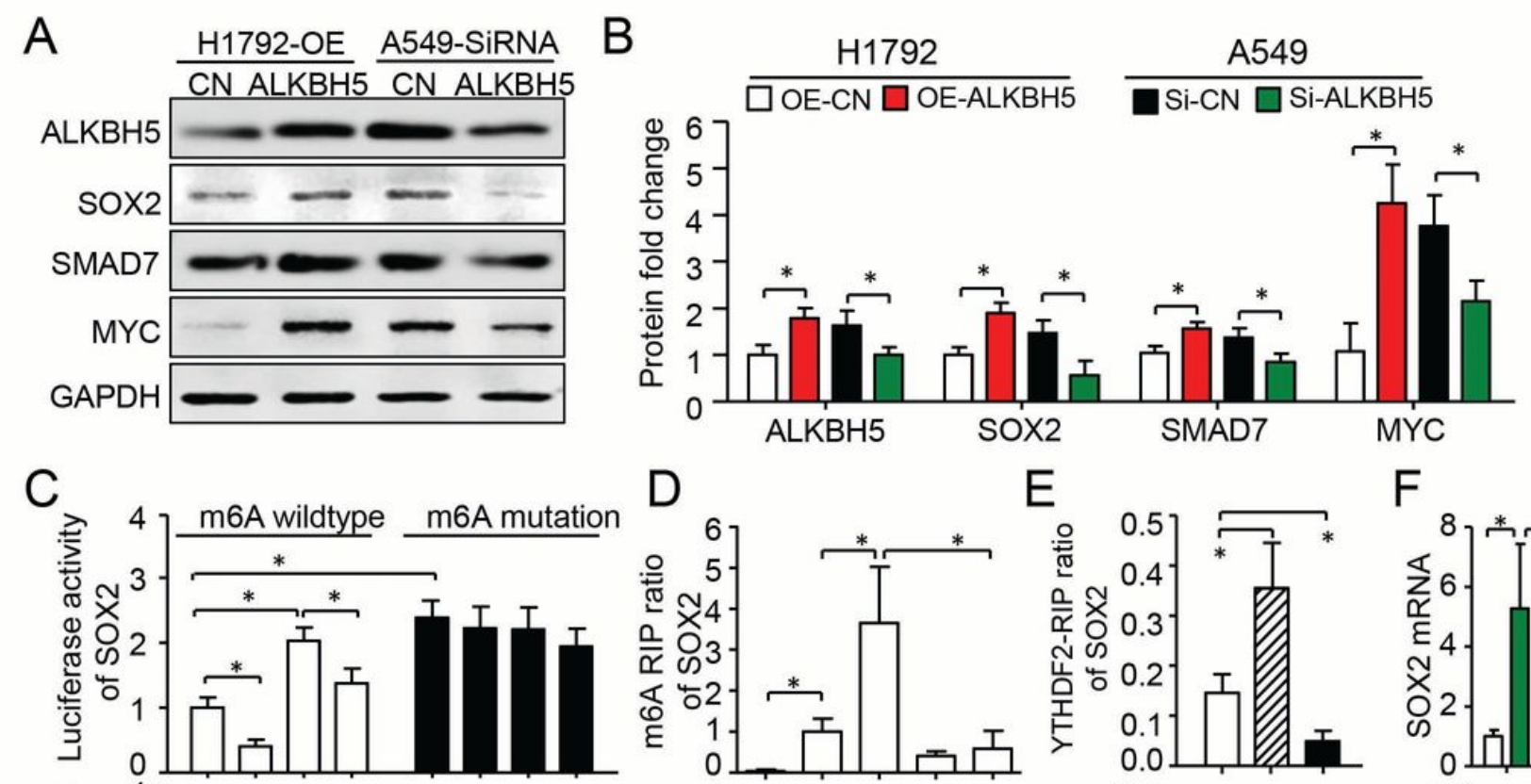

E
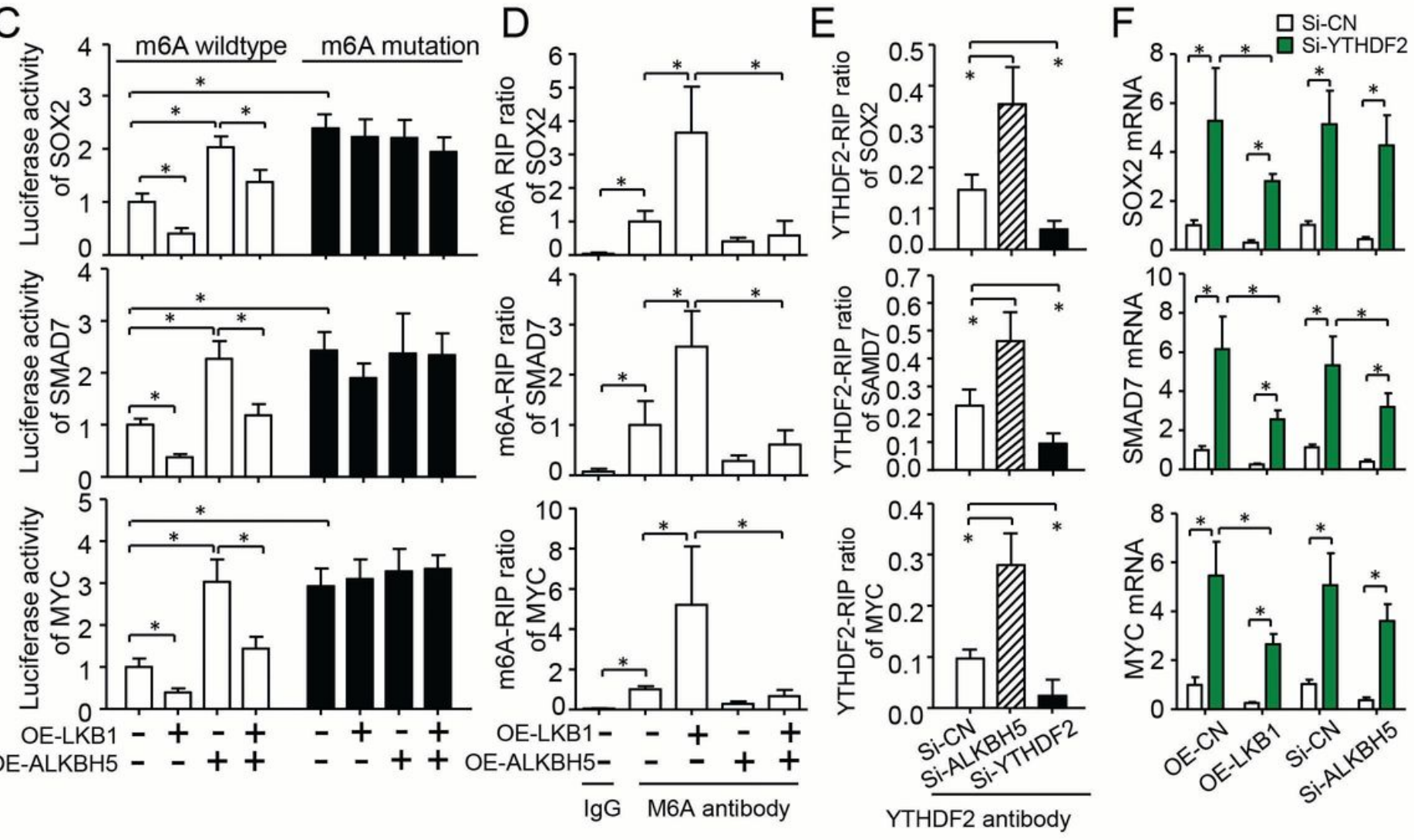

OE-ALKBH5

IgG M6A antibody

YTHDF2 antibody

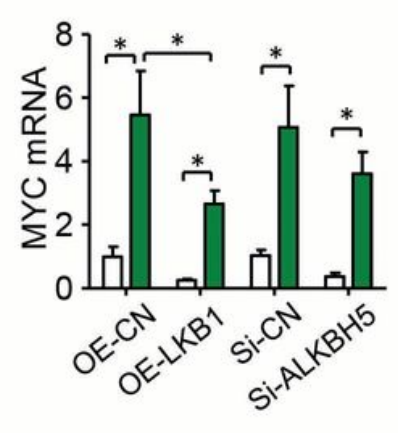

Figure 6

ALKBH5 erases m6A modification on SOX2, SMAD7 and MYC mRNA to increase their stability and expression. (A, B) Representative western blotting and quantification of SOX2, SMAD7, MYC and ALKBH5 protein. Data as mean $\pm S D(n=4)$. (C) Mutation of m6A site or LKB1 over-expression released the SOX2, 
SMAD7 and MYC gene post-transcriptional repression by ALKBH5 in A549 cells $(n=4)$. (D) m6A-RIP analysis demonstrated that SOX2, SMAD7 and MYC were subjected to ALKBH5-mediated m6A modifications $(n=3)$. (E) YTHDF2-RIP-qPCR shown that YTHDF2 could occupy m6A sites of SOX2, SMAD7 and MYC, which was mediated by ALKBH5 ( $n=4)$. (F) qRT-PCR was performed to indicate that knockdown of YTHDF2 significantly up-regulated SOX2, SMAD7 and MYC gene mRNA expression at basal level, LKB1 over-expressed or ALKBH5 silenced A549 cells $(n=3)$. Data as mean $\pm S D$. ${ }^{*} P<0.05$ by two-way ANOVA followed by Bonferroni multiple comparison post hoc test for B-F. Si-CN, Si-RNA-A. OECN, OE-c-Flag pcDNA3. OE, over-expression.
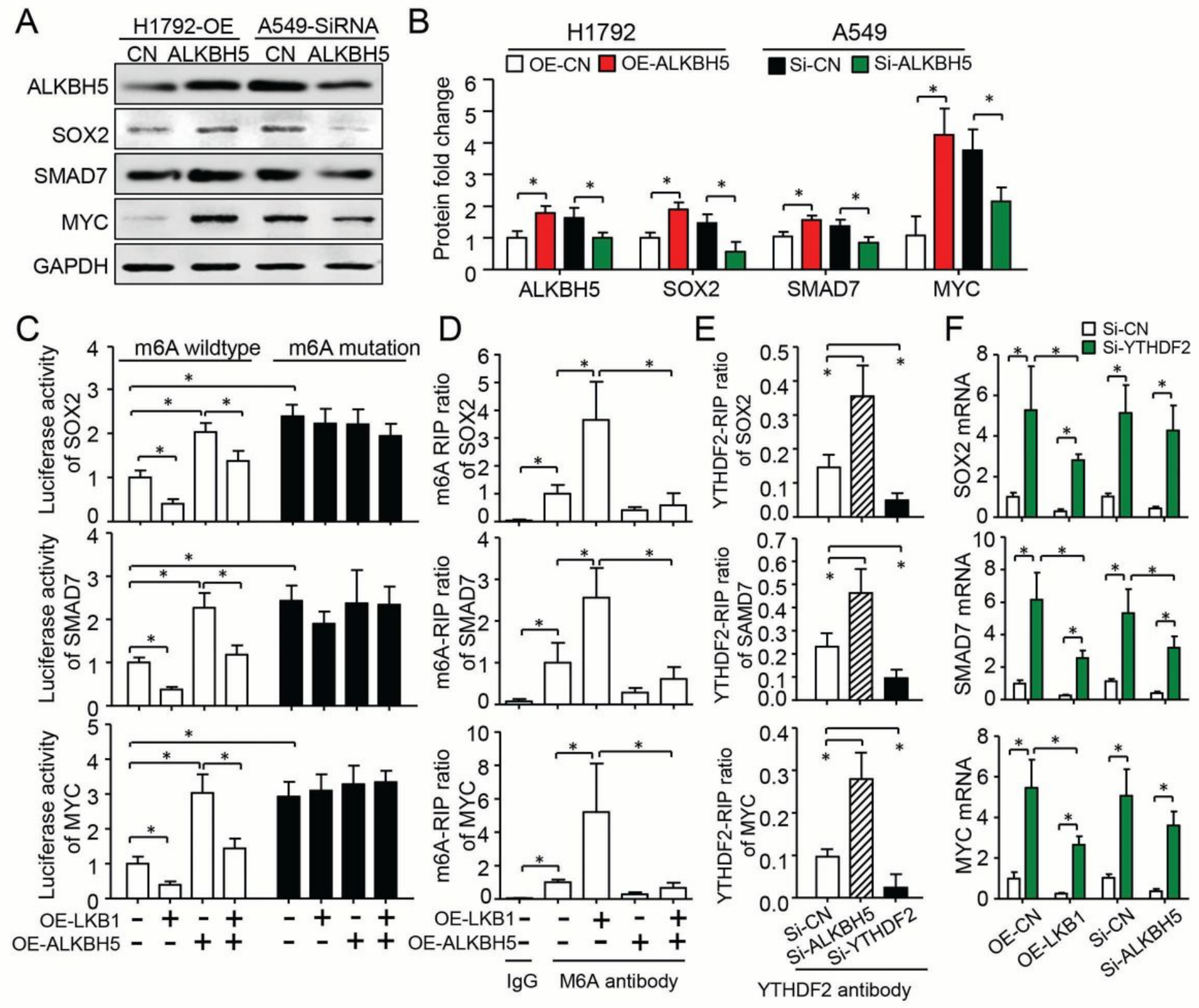

OE-ALKBH5

IgG M6A antibody

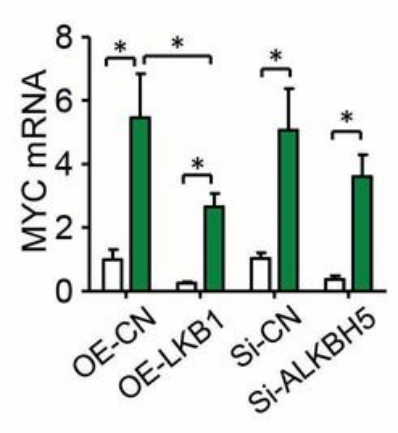

Figure 6

ALKBH5 erases m6A modification on SOX2, SMAD7 and MYC mRNA to increase their stability and expression. (A, B) Representative western blotting and quantification of SOX2, SMAD7, MYC and ALKBH5 protein. Data as mean $\pm S D(n=4)$. (C) Mutation of m6A site or LKB1 over-expression released the SOX2, SMAD7 and MYC gene post-transcriptional repression by ALKBH5 in A549 cells ( $n=4)$. (D) m6A-RIP 
analysis demonstrated that SOX2, SMAD7 and MYC were subjected to ALKBH5-mediated m6A modifications $(n=3)$. (E) YTHDF2-RIP-qPCR shown that YTHDF2 could occupy m6A sites of SOX2, SMAD7 and MYC, which was mediated by ALKBH5 $(n=4)$. (F) qRT-PCR was performed to indicate that knockdown of YTHDF2 significantly up-regulated SOX2, SMAD7 and MYC gene mRNA expression at basal level, LKB1 over-expressed or ALKBH5 silenced A549 cells $(n=3)$. Data as mean $\pm S D$. ${ }^{*} P<0.05$ by two-way ANOVA followed by Bonferroni multiple comparison post hoc test for B-F. Si-CN, Si-RNA-A. OECN, OE-c-Flag pcDNA3. OE, over-expression.

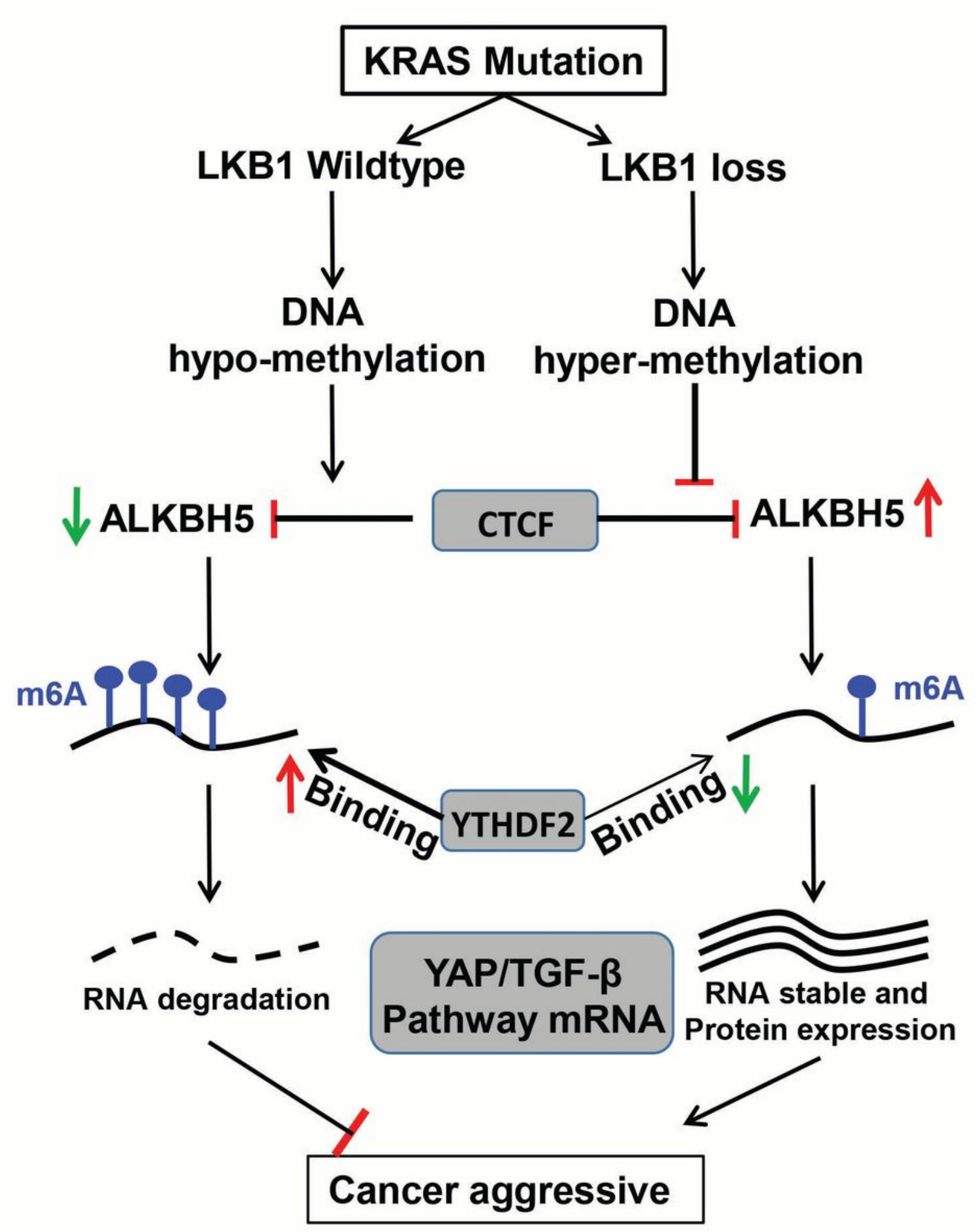


Figure 7

Proposed model for LKB1-mediated m6A modification in KRAS mutated lung cancer progression. Loss of LKB1-induced DNA hypermethylation, which prevents CTCF binding on the ALKBH5 gene promoter, maintains ALKBH5 expression, and further represses global RNA methylation. Oncogenic SMAD7, SOX2, and MYC are crucial targets of m6A meditated by LKB1 deficiency, and are involved in KRAS mutation lung cancer progression.

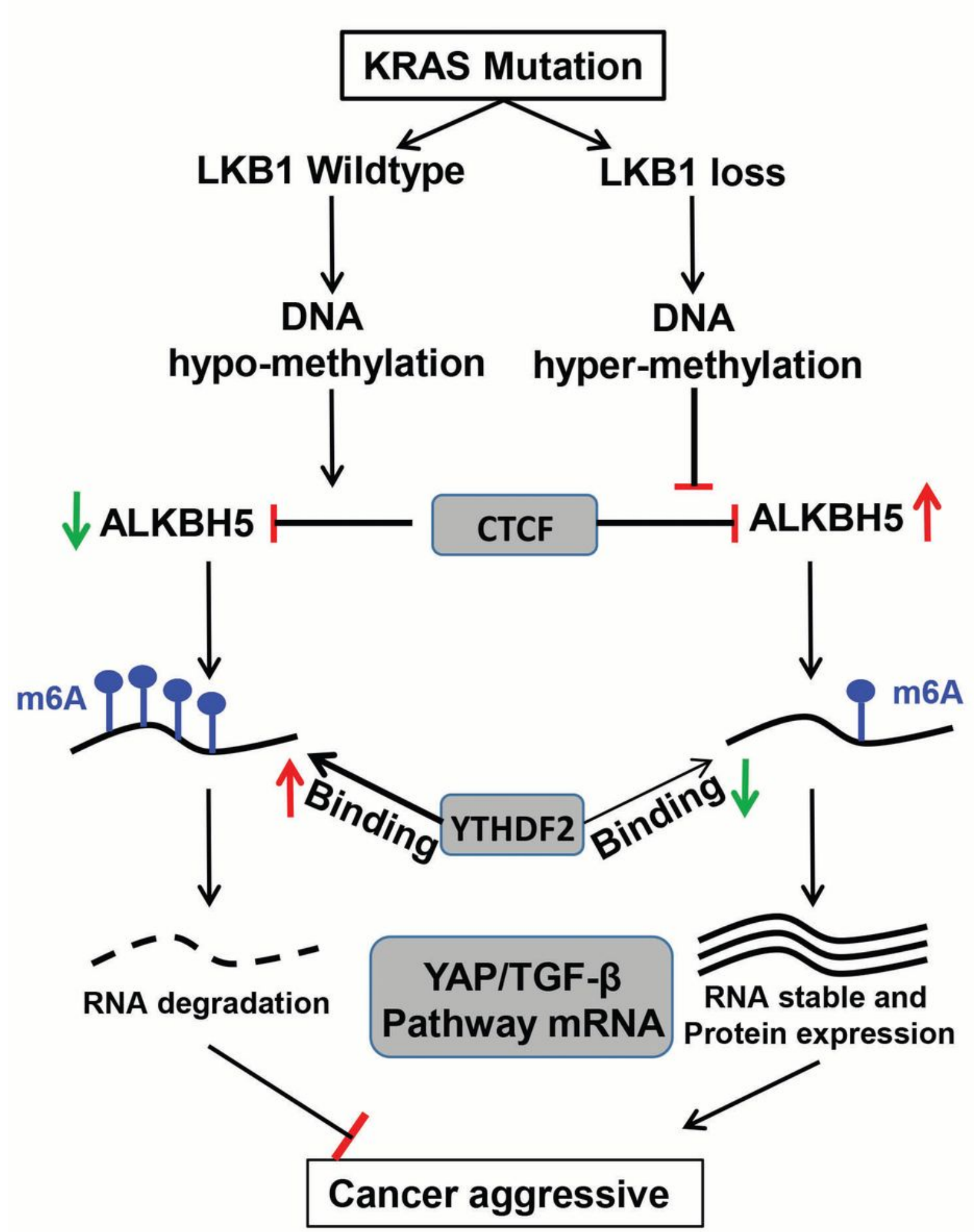




\section{Figure 7}

Proposed model for LKB1-mediated m6A modification in KRAS mutated lung cancer progression. Loss of LKB1-induced DNA hypermethylation, which prevents CTCF binding on the ALKBH5 gene promoter, maintains ALKBH5 expression, and further represses global RNA methylation. Oncogenic SMAD7, SOX2, and MYC are crucial targets of m6A meditated by LKB1 deficiency, and are involved in KRAS mutation lung cancer progression.

\section{Supplementary Files}

This is a list of supplementary files associated with this preprint. Click to download.

- SupplementaryFigure19TableS12.pdf

- SupplementaryFigure19TableS12.pdf 